\title{
TRANSLATION TO AND FRO OVER KAC-MOODY ALGEBRAS
}

\author{
WAYNE NEIDHARDT
}

\begin{abstract}
Over a (not necessarily symmetrizable) Kac-Moody algebra, we define translation functors in both the dominant and the antidominant directions, and prove an adjoint-like property relating the two translation functors. Using this property, we show that for any $x$ and $y$ in the Weyl group, the numbers $\operatorname{dim} \operatorname{Ext}^{n}(M(x \cdot \lambda), L(y \cdot \lambda))$ relating Verma modules and irreducible modules do not depend on the choice of dominant integral weight $\lambda$. We then define operators of coherent continuation and polynomials analogous to the Kazhdan-Lusztig polynomials and study some of their properties.
\end{abstract}

1. Introduction. Translation functors over (not necessarily symmetrizable) Kac-Moody algebras were introduced in [11], with certain restrictions on which translations were allowed, using the idea of local projective resolutions, also introduced in [11]. These translation functors were a generalization of those introduced, in the case of a finite dimensional algebra, by Jantzen in [6]. The main restriction we placed on translations was that in order to translate to, say, the weight $\lambda^{\prime}$ from the weight $\lambda^{\prime \prime}$, we required that $\lambda^{\prime}-\lambda^{\prime \prime}$ be dominant integral, since part of the definition involves taking the tensor product with the irreducible module $L\left(\lambda^{\prime}-\lambda^{\prime \prime}\right)$ of highest weight $\lambda^{\prime}-\lambda^{\prime \prime}$.

In the present paper, we show how to translate back to $\lambda^{\prime \prime}$ from $\lambda^{\prime}$. The main difficulty is that the irreducible module of extreme weight $\lambda^{\prime \prime}-\lambda^{\prime}$ is a lowest weight module, so that in general when one tensors, for example, a Verma module with this lowest weight module, the result no longer has finite dimensional weight spaces, nor is the set of weights bounded above. Thus, the usual techniques used in the category $\mathscr{O}$ or in similar categories are not sufficient to deal with this problem. We therefore are led to introduce the notion of modules with reverse Verma series, and, after proving some of their properties, deal with the situation of a Verma module tensored with a lowest weight module. Once this difficulty has been overcome, we are able to define the translation functors from $\lambda^{\prime}$ to $\lambda^{\prime \prime}$ in the situation described above, using local projective resolutions as before. 
After defining translation functors in both directions, we apply them in certain situations to obtain instances of a translation principle, which says roughly that "whatever happens in one Weyl group orbit happens in another, provided the two orbits have representatives in the same facette." In particular, we show for any $n \geq 0$ that $\operatorname{dim} \operatorname{Ext}^{n}(M(x \cdot \lambda), L(y \cdot \lambda))$, where $M(\mu)$ denotes the Verma module of highest weight $\mu$, depends only on the elements $x$ and $y$ of the Weyl group and on the facette of $\lambda$, but not on the particular choice of $\lambda$ within that facette. We also apply the functors to the situation of translation from a chamber to a wall and back again, thus obtaining the operators of coherent continuation. Finally, we apply the result on Ext mentioned above to define polynomials $\bar{P}_{x, y}$ for each $x$ and $y$ in the Weyl group. These are analogous to the Kazhdan-Lusztig polynomials introduced in [8], except that we use a dominant integral weight rather than an antidominant integral weight (so that in the finite dimensional case we have $\bar{P}_{x, y}=P_{x w_{0}, y w_{0}}$, where $w_{0}$ is the longest element of the Weyl group).

The paper is organized as follows. Section 2 gives the basic terminology and notation associated with Kac-Moody algebras. In $\S 3$ we recall the local projective resolutions introduced in [11], but in slightly more generality. These resolutions allow us to do homological algebra in certain categories of modules which do not have enough projectives, and will be used to define translation functors and other functors. We study reverse Verma series as well as Verma series in $\S 4$, and apply the results of $\S \S 3$ and 4 in $\S 5$ to define translation functors from one Weyl group orbit to another. Section 6 is devoted to the construction of a spectral sequence converging to the translation functors for the case of translation in one direction. This spectral sequence is used in $\S 7$ to prove an adjoint-like property relating the two translation functors which translate back and forth between two Weyl group orbits. This adjoint-like property is the key to our result on Ext, mentioned above, which is proved in $\S 8$. Operators of coherent continuation are defined in $\S 9$, and their basic properties are derived. In $\S 10$ we introduce the polynomials $\bar{P}_{x, y}$ and prove some of their properties.

2. Definitions and notation. We give in this section the basic definitions and notation associated with Kac-Moody algebras and their representations. We also introduce certain categories of modules which will be used in the sequel, along with certain types of filtrations on those modules. 
Let $A=\left(A_{i j}\right)$ be an $l \times l$ generalized Cartan matrix (GCM), so that $A_{i i}=2$ for all $i, A_{i j}$ is a nonpositive integer for $i \neq j$, and $A_{i j}=0$ if and only if $A_{j i}=0$. Let $K$ be a field of characteristic zero, and let $\mathfrak{g}$ be any Lie algebra over $K$ satisfying

(1) $\mathfrak{g}$ is generated by a finite dimensional abelian subalgebra $\mathfrak{h}$, called the Cartan subalgebra, and elements $e_{1}, \ldots, e_{l}, f_{1}, \ldots, f_{l}$, called simple root vectors and negative simple root vectors, respectively.

(2) There are linearly independent sets $\left\{h_{1}, \ldots, h_{l}\right\}$ in $\mathfrak{h}$ and $\left\{\alpha_{1}, \ldots\right.$, $\left.\alpha_{l}\right\}$ in $\mathfrak{h}^{*}$, the dual vector space of $\mathfrak{h}$, such that $A_{i j}=\alpha_{j}\left(h_{i}\right)$ for all $i$ and $j$. The $\alpha_{j}$ 's are called the simple roots, and the $h_{i}$ 's are called the simple coroots.

(3) $\left[e_{i}, f_{j}\right]=\delta_{l j} h_{i}$ for all $i, j=1, \ldots, l$.

(4) $\left[h, e_{i}\right]=\alpha_{i}(h) e_{i}$ and $\left[h, f_{i}\right]=-\alpha_{i}(h) f_{i}$ for all $h \in \mathfrak{h}$ and all $i=1, \ldots, l$.

(5) $\left(\operatorname{ad} e_{i}\right)^{-A_{i \jmath}+1}\left(e_{j}\right)=0=\left(\operatorname{ad} f_{i}\right)^{-A_{i j}+1}\left(f_{j}\right)$ for all $i \neq j$.

(6) There is an involutive antiautomorphism $\sigma: \mathfrak{g} \rightarrow \mathfrak{g}$ such that $\sigma\left(e_{i}\right)=f_{i}$ for all $i=1, \ldots, l$, and $\sigma(h)=h$ for all $h \in \mathfrak{h}$.

For any GCM $A$, such an algebra $\mathfrak{g}$ exists and is called a Kac-Moody algebra defined by $A$. These objects were introduced in [7 and 9].

Denote by $\mathfrak{n}^{+}$(respectively, $\mathfrak{n}^{-}$) the subalgebra of $\mathfrak{g}$ generated by $\left\{e_{1}, \ldots, e_{l}\right\}$ (respectively, $\left\{f_{1}, \ldots, f_{l}\right\}$ ), and set $\mathfrak{b}=\mathfrak{h} \oplus \mathfrak{n}^{+}$, called the Borel subalgebra of $\mathfrak{g}$. For each $i=1, \ldots, l$, let $\mathfrak{a}_{i}=K h_{i} \oplus K e_{i} \oplus K f_{i}$. It is easy to see from the relations that $\mathfrak{a}_{i}$ is a subalgebra of $\mathfrak{g}$ isomorphic to $\operatorname{sl}(2, K)$.

For any $\mathfrak{h}$-module $M$ and any $\lambda \in \mathfrak{h}^{*}$, let $M_{\lambda}=\{m \in M \mid h \cdot m=$ $\lambda(h) m$ for all $h \in \mathfrak{h}\}$, called the $\lambda$-weight space of $M$; if $M_{\lambda} \neq 0$ we call $\lambda$ a weight of $M$. In case $M$ is an $\mathfrak{h}$-module satisfying $M=$ $\bigoplus_{\lambda \in h^{*}} M_{\lambda}$, we call $M$ a weight module, and we write $\Pi(M)$ for the set of weights of $M$. If in addition each $M_{\lambda}$ is finite dimensional, we define the character of $M$ to be $\operatorname{ch} M=\sum_{\mu \in \mathfrak{h}^{*}}\left(\operatorname{dim} M_{\mu}\right) e^{\mu}$, where the $e^{\mu}$ 's are formal exponentials. Note that this discussion of weights and weight modules applies as well to $\mathfrak{g}$-modules and $\mathfrak{b}$-modules, which may be viewed as $\mathfrak{h}$-modules by restriction. In particular, for the adjoint representation of $\mathfrak{g}$, we let $\Delta=\left\{\alpha \in \mathfrak{h}^{*} \mid \mathfrak{g}_{\alpha} \neq 0\right.$ and $\left.\alpha \neq 0\right\}$ and call $\Delta$ the set of roots of $\mathfrak{g}$.

In the sequel, whenever $M$ and $N$ are $\mathfrak{g}$-modules which are weight modules, we will write $\operatorname{Hom}(M, N)$ for the set of $\mathfrak{g}$-module maps from $M$ to $N$, and $\operatorname{Ext}(M, N)$ for the set of equivalence classes of extensions of $M$ by $N$ in the category of weight modules. 
We define an ordering on $\mathfrak{h}^{*}$ by letting $\mu \leq \lambda$ if $\lambda-\mu=\sum_{i=1}^{l} k_{i} \alpha_{i}$, where $k_{i} \in \mathbb{Z}_{+}$, the set of nonnegative integers. We set $\Delta^{+}=$ $\{\alpha \in \Delta \mid \alpha>0\}$, called the set of positive roots, and $\Delta^{-}=-\Delta^{+}=$ $\{\alpha \in \Delta \mid \alpha<0\}$. Also, let $P=\left\{\lambda \in \mathfrak{h}^{*} \mid \lambda\left(h_{i}\right) \in \mathbb{Z}\right.$ for all $\left.i\right\}$, called the set of integral weights, and let $P^{+}=\left\{\lambda \in \mathfrak{h}^{*} \mid \lambda\left(h_{i}\right) \in \mathbb{Z}_{+}\right.$for all $\left.i\right\}$, called the set of dominant integral weights.

For any $\lambda \in \mathfrak{h}^{*}$, we denote by $C(\lambda)$ the full subcategory of $\mathfrak{g}$-modules $M$ such that $M$ is a weight module with finite dimensional weight spaces and such that $\Pi(M) \subseteq\{\mu \mid \mu \leq \lambda\}$.

Suppose $M$ is a $\mathfrak{g}$-module. If $v \in M_{\lambda}$ is a nonzero vector satisfying $\eta^{+} \cdot v=0$, we call $v$ a maximal vector. If in addition $M=U(\mathfrak{g}) v$, where $U(-)$ denotes the universal enveloping algebra functor, we call $v$ a highest weight vector and call $M$ a highest weight module of weight $\lambda$. Every highest weight module is a weight module.

Let $\lambda \in \mathfrak{h}^{*}$, and denote by $K(\lambda)$ the one-dimensional $\mathfrak{b}$-module whose underlying vector space is $K$ and whose $\mathfrak{g}$-module structure is given by $(h+x) \cdot k=\lambda(h) k$ for all $h \in \mathfrak{h}, x \in \mathfrak{n}^{+}$, and $k \in K$. The Verma module of weight $\lambda$ is then the induced $\mathfrak{g}$-module $M(\lambda)=U(\mathfrak{g}) \otimes_{U(\mathfrak{b})} K(\lambda)$. It is well known that $M(\lambda)$ is the universal highest weight module of weight $\lambda$, and that it has a unique irreducible quotient, denoted by $L(\lambda)$. Similarly, we may define lowest weight modules, and let $W(\lambda)$ be the universal lowest weight module of weight $\lambda$ and $\Gamma(\lambda)$ its unique irreducible quotient. (These may be thought of as "upsidedown" versions of $M(\lambda)$ and $L(\lambda)$, and hence the choice of notation.)

For each $i=1, \ldots, l$, define the linear involution $r_{i}: \mathfrak{h}^{*} \rightarrow \mathfrak{h}^{*}$ by $r_{i}(\lambda)=\lambda-\lambda\left(h_{i}\right) \alpha_{i}$ for all $\lambda \in \mathfrak{h}^{*}$. The Weyl group $W$ is then defined to be the subgroup of $\mathrm{GL}\left(\mathfrak{h}^{*}\right)$ generated by $\left\{r_{1}, \ldots, r_{l}\right\}$. For any $w \in W$, we define the length of $w$, denoted $l(w)$, to be the smallest $n$ such that $w$ may be written in the form $w=r_{i_{1}} \ldots r_{i_{n}}$ for some $i_{1}, \ldots, i_{n} \in$ $\{1, \ldots, l\}$. Let $\rho \in \mathfrak{h}^{*}$ be a fixed element such that $\rho\left(h_{i}\right)=1$ for all $i$, and define the dot action of $W$ on $\mathfrak{h}^{*}$ by $w \cdot \lambda=w(\lambda+\rho)-\rho$ for all $w \in W, \lambda \in \mathfrak{h}^{*}$.

Let $\Pi=\left\{\alpha_{1}, \ldots, \alpha_{l}\right\}$, so that $\Pi$ is the set of simple roots, and define $\Delta_{R}=W \Pi$, called the set of real roots, $\Delta_{R}^{+}=\Delta_{R} \cap \Delta^{+}$, and $\Delta_{R}^{-}=$ $\Delta_{R} \cap \Delta^{-}$. For any $\alpha \in \Delta_{R}^{+}$we define $r_{\alpha} \in W$ by $r_{\alpha}=w r_{i} w^{-1}$, where $\alpha=w \alpha_{i}$ with $w \in W$. This can be shown to be independent of the choices of $w$ and $\alpha_{i}$.

We now define three types of filtrations of modules which will be used in the sequel. 
Definition 2.1 ([4]). Let $M$ be a weight module, all of whose weight spaces are finite dimensional, and let $\mu \in \mathfrak{h}^{*}$. By a local composition series (LCS) for $M$ at $\mu$, we mean a finite sequence $0=M_{0} \subset M_{1} \subset \cdots \subset M_{n}=M$ of submodules of $M$ such that each factor $F_{i}=M_{i} / M_{i-1}$ satisfies either $\Pi\left(F_{i}\right) \cap\{\nu \mid \nu \geq \mu\}=\varnothing$ or $F_{i} \cong L\left(\mu_{i}\right)$ for some $\mu_{i} \geq \mu$. We write $(M: L(\mu))$ for the number of $i$ such that $\mu_{i}=\mu$. If $(M: L(\mu)) \neq 0$, we call $L(\mu)$ a composition factor of $M$.

RemarK. In [4, Proposition 4.2] it is shown that if $\sum_{\nu \geq \mu} \operatorname{dim} M_{\nu}$ is finite, then $M$ has an LCS at $\mu$. In particular, such series always exist for modules which are objects in categories of the form $C(\lambda)$. It is also shown in [4] that the number $(M: L(\mu))$ does not depend on the choice of LCS.

Definition 2.2. Let $M$ be a weight module. By a Verma series (VS) for $M$, we mean a sequence (possibly finite) $0=M_{0} \subset M_{1} \subset$ ... of submodules of $M$ such that (i) $M=\bigcup_{i \geq 0} M_{i}$, (ii) each factor $F_{i}=M_{i} / M_{i-1} \cong M\left(\lambda_{i}\right)$ for some $\lambda_{i} \in \mathfrak{h}^{*}$, and (iii) for any $\mu \in \mathfrak{h}^{*}$, $\left\{i \mid \lambda_{i} \geq \mu\right\}$ is a finite set. In case $M$ has such a VS and $\mu \in \mathfrak{h}^{*}$, we write $(M: M(\mu))$ for the number of $i$ such that $\lambda_{i}=\mu$.

REMARK. Condition (iii) could be replaced by the requirement that all weight spaces of $M$ be finite dimensional. We prefer the above definition, however, because of its analogy with that of reverse Verma series, given below, in which the weight spaces are almost never finite dimensional. Also, the numbers $(M: M(\mu))$ can be shown to be independent of the choice of VS, if one exists, by character considerations.

Definition 2.3. Let $M$ be a weight module. By a reverse Verma series (RVS) for $M$, we mean a sequence (possibly finite) $M=M_{0} \supset$ $M_{1} \supset$... such that (i) $\bigcap_{i>0} M_{i}=0$, (ii) each factor $F_{i}=M_{i-1} / M_{i} \cong$ $M\left(\lambda_{i}\right)$ for some $\lambda_{i} \in \mathfrak{h}^{*}$, (iii) for any $\mu \in \mathfrak{h}^{*},\left\{i \mid \lambda_{i} \leq \mu\right\}$ is a finite set, and (iv) there exist vectors $v_{i} \in M_{\lambda_{1}}$ such that $v_{i}+M_{i}$ is a highest weight vector for $F_{i}$ and such that $M_{i}=\sum_{j>i} U\left(\mathfrak{n}^{-}\right) v_{j}$ for each $i=0,1, \ldots$

REMARK. In the case of a VS, any choice of weight vectors which represent highest weight vectors of the factors satisfies the analogue of condition (iv) in the definition of RVS. The difference in the behaviors of direct and inverse limits is what forces us to specify this condition in the case of an RVS.

Modules with RVS, as well as those with VS, will be studied in depth in $\S 4$. Observe that a finite RVS is the same as a finite VS. In the infinite case, however, they are quite different. 
3. Projectives and local projective resolutions. We recall the definition and basic properties of local projective resolutions, which were introduced in [11], and prove some of their homological properties in more generality than that of [11]. These are essentially a tool for doing homological algebra in categories which do not have enough projectives, specifically the categories $C(\lambda)$ defined in the previous section. We will use them in defining translation functors and other functors.

Throughout this section, let $\lambda \in \mathfrak{h}^{*}$ be fixed. We begin with the basic properties of the projectives in $C(\lambda)$ introduced in [13].

Proposition 3.1 [13, Propositions 4.5, 4.7, 4.8 and 5.3, Lemma 4.12, Corollary 4.13 and Theorem 6.2]. For each irreducible object $L(\mu)$ in $C(\lambda)$, there is a unique (up to isomorphism) finitely generated indecomposable projective object $I^{\lambda}(\mu)$ in $C(\lambda)$ which maps onto $L(\mu)$. Conversely, every finitely generated indecomposable projective object in $C(\lambda)$ has a unique irreducible quotient, so that $L(\mu) \leftrightarrow I^{\lambda}(\mu)$ gives a one-to-one correspondence between the irreducible objects in $C(\lambda)$ and the finitely generated indecomposable projective objects in $C(\lambda)$. Furthermore, $I^{\lambda}(\mu)$ has a finite VS with $M(\mu)$ as the top factor, and $\left(I^{\lambda}(\mu): M(\nu)\right)=(M(\nu): L(\mu))$ for all $\mu, \nu \leq \lambda$. Finally, for any $M \in$ Ob $C(\lambda)$ we have $(M: L(\mu))=\operatorname{dim} \operatorname{Hom}\left(I^{\lambda}(\mu), M\right)$.

Since $C(\lambda)$ does not have enough projectives, projective resolutions cannot in general be constructed. Thus we rely on the following concept.

Definition 3.2 ([11, Definition 3.6]). Let $\mu \in \mathfrak{h}^{*}$ and $M \in \mathrm{Ob} C(\lambda)$. A local projective resolution of $M$ at $\mu$ is a complex

$$
\cdots \rightarrow P_{k} \rightarrow \cdots \rightarrow P_{0} \rightarrow M \rightarrow 0
$$

in $C(\lambda)$, where each $P_{k}$ is a finite direct sum of various $I^{\lambda}(\nu)$ with $\nu \geq \mu$, and such that for any $\chi \geq \mu$, the restriction

$$
\cdots \rightarrow\left(P_{k}\right)_{\chi} \rightarrow \cdots \rightarrow\left(P_{0}\right)_{\chi} \rightarrow M_{\chi} \rightarrow 0
$$

to the $\chi$-weight spaces is exact.

Proposition 3.3 [11, Proposition 3.7]. For any $\mu \in \mathfrak{h}^{*}$ and any $M \in \mathrm{Ob} C(\lambda)$, there exists a local projective resolution of $M$ at $\mu$.

Proposition 3.4 [11, Proposition 3.8]. Let $M, N \in \mathrm{Ob} C(\lambda)$, and let $\mu_{1} \geq \mu_{2}$. Suppose

$\left(P_{*}\right): \quad \cdots \rightarrow P_{k} \rightarrow \cdots \rightarrow P_{0} \rightarrow M \rightarrow 0$ 
is a local projective resolution of $M$ at $\mu_{1}$, and

$$
\left(Q_{*}\right): \quad \cdots \rightarrow Q_{k} \rightarrow \cdots \rightarrow Q_{0} \rightarrow N \rightarrow 0
$$

is a local projective resolution of $N$ at $\mu_{2}$. Then for any $\phi \in \operatorname{Hom}(M, N)$ there is a morphism of complexes $\bar{\phi}:\left(P_{*}\right) \rightarrow\left(Q_{*}\right)$ over $\phi$, and the morphism $\bar{\phi}$ is unique up to homotopy.

Proposition 3.5 [11, Proposition 3.10]. Let $\mu \in \mathfrak{h}^{*}$, and suppose

$$
0 \rightarrow M^{\prime} \stackrel{\phi}{\rightarrow} M \stackrel{\psi}{\rightarrow} M^{\prime \prime} \rightarrow 0
$$

is a sequence in $C(\lambda)$ whose restriction to the $\chi$-weight spaces is exact for every $\chi \geq \mu$. Suppose also that

$\left(P_{*}^{\prime}\right)$ :

$$
\cdots \rightarrow P_{k}^{\prime} \rightarrow \cdots \rightarrow P_{0}^{\prime} \rightarrow M^{\prime} \rightarrow 0
$$

and

$\left(P_{*}^{\prime \prime}\right)$ :

$$
\cdots \rightarrow P_{k}^{\prime \prime} \rightarrow \cdots \rightarrow P_{0}^{\prime \prime} \rightarrow M^{\prime \prime} \rightarrow 0
$$

are local projective resolutions of $M^{\prime}$ and $M^{\prime \prime}$, respectively, at $\mu$. Then, setting $P_{k}=P_{k}^{\prime} \oplus P_{k}^{\prime \prime}$ for each $k$, there exist maps $P_{k} \rightarrow P_{k-1}(k=$ $1,2, \ldots)$ and $P_{0} \rightarrow M$ such that

$\left(P_{*}\right)$ :

$$
\cdots \rightarrow P_{k} \rightarrow \cdots \rightarrow P_{0} \rightarrow M \rightarrow 0
$$

is a local projective resolution of $M$ at $\mu$, and such that the canonical injections give a morphism of complexes $\left(P_{*}^{\prime}\right) \rightarrow\left(P_{*}\right)$ over $\phi$ and the canonical projections give a morphism of complexes $\left(P_{*}\right) \rightarrow\left(P_{*}^{\prime \prime}\right)$ over $\psi$.

We now use these properties of local projective resolutions to define, in somewhat more generality than that of [11], the concepts which may be used in doing homological algebra in $C(\lambda)$.

Definition 3.6. Let $C$ be an abelian category with direct limits, and let $F$ be an additive functor from the full subcategory of $C(\lambda)$ whose objects are finite direct sums of various $I^{\lambda}(\nu)$ to the category $C$. For any $\mu \in \mathfrak{h}^{*}$ and any $q=0,1, \ldots$, we define the $q$ th $\mu$-local left derived functor of $F$, denoted $L_{q, \mu} F$, from $C(\lambda)$ to $C$ as follows. For any $M \in \mathrm{Ob} C(\lambda)$, we define $\left(L_{q, \mu} F\right) M$ to be the $q$ th homology $H_{q}\left(F P_{*}\right)$ of the complex $\left(F P_{*}\right)$ :

$$
\cdots \rightarrow F P_{k} \rightarrow \cdots \rightarrow F P_{0} \rightarrow 0,
$$

where

$\left(P_{*}\right)$ :

$$
\cdots \rightarrow P_{k} \rightarrow \cdots \rightarrow P_{0} \rightarrow M \rightarrow 0
$$


is a local projective resolution of $M$ at $\mu$. Furthermore, if $\phi: M \rightarrow N$ is a morphism in $C(\lambda)$, we define $\left(L_{q, \mu} F\right) \phi$ to be the map induced on homology by $F \bar{\phi}_{q}$, where $\bar{\phi}:\left(P_{*}\right) \rightarrow\left(Q_{*}\right)$ is a morphism of projective resolutions of $M$ and $N$, respectively, at $\mu$, lying over $\phi$. Finally, define the left locally derived functors $L_{q} F$ from $C(\lambda)$ to $C$ by

$$
\left(L_{q} F\right) M=\underset{\mu}{\stackrel{\lim }{\longrightarrow}}\left(L_{q, \mu} F\right) M \quad \text { and } \quad\left(L_{q} F\right) \phi=\underset{\mu}{\lim }\left(L_{q, \mu} F\right) \phi .
$$

REMARK. By Proposition 3.4, $\left(L_{q, \mu} F\right) M$ is well defined, taking $\phi$ to be the identity on $M$, and $\left(L_{q, \mu} F\right) \phi$ is well defined by the uniqueness up to homotopy. We also obtain unique maps $\left(L_{q, \mu_{1}} F\right) M \rightarrow$ $\left(L_{q, \mu_{2}} F\right) M$ whenever $\mu_{1} \geq \mu_{2}$ by Proposition 3.4 , so that the direct limit in the above definition makes sense.

Using Proposition 3.5 and the exactness of direct limits, we easily obtain the following.

THEOREM 3.7. Let $C$ be an abelian category with direct limits and let $F$ be an additive functor from the full subcategory of $C(\lambda)$ whose objects are finite direct sums of various $I^{\lambda}(\nu)$ to the category $C$. Let

$$
0 \rightarrow M^{\prime} \rightarrow M \rightarrow M^{\prime \prime} \rightarrow 0
$$

be an exact sequence in $C(\lambda)$. Then, for any $\mu \in \mathfrak{h}^{*}$, there is a long exact sequence in homology

$$
\begin{aligned}
\cdots & \rightarrow\left(L_{q, \mu} F\right) M^{\prime} \rightarrow\left(L_{q, \mu} F\right) M \rightarrow\left(L_{q, \mu} F\right) M^{\prime \prime} \\
& \rightarrow\left(L_{q-1, \mu} F\right) M^{\prime} \rightarrow \cdots \rightarrow\left(L_{0, \mu} F\right) M^{\prime \prime} \rightarrow 0,
\end{aligned}
$$

and, passing to the direct limits, there is a long exact sequence

$$
\begin{aligned}
\cdots & \rightarrow\left(L_{q} F\right) M^{\prime} \rightarrow\left(L_{q} F\right) M \rightarrow\left(L_{q} F\right) M^{\prime \prime} \\
& \rightarrow\left(L_{q-1} F\right) M^{\prime} \rightarrow \cdots \rightarrow\left(L_{0} F\right) M^{\prime \prime} \rightarrow 0 .
\end{aligned}
$$

In the remainder of this section, we construct some projective resolutions of modules with finite VS.

Definition 3.8. Let $\mu \leq \lambda$. Then we define $S^{\lambda}(\mu)$ to be the kernel of any nonzero $\phi \in \operatorname{Hom}\left(I^{\lambda}(\mu), M(\mu)\right)$.

Remark. Since $\operatorname{dim} \operatorname{Hom}\left(I^{\lambda}(\mu), M(\mu)\right)=(M(\mu): L(\mu))=1$, the module $S^{\lambda}(\mu)$ does not depend on the choice of nonzero map. 
Proposition 3.9. Let $\mu \leq \lambda$. Then $S^{\lambda}(\mu)$ has a finite $V S$, each of whose factors $M(\nu)$ satisfies $\nu>\mu$. Furthermore, for any $\mu<\nu \leq \lambda$, we have

$$
\left(S^{\lambda}(\mu): M(\nu)\right)=\left(I^{\lambda}(\mu): M(\nu)\right)=(M(\nu): L(\mu)) .
$$

Proof. By Proposition 3.1, we may take a finite VS $0=M_{0} \subset$ $M_{1} \subset \cdots \subset M_{n-1} \subset M_{n}=I^{\lambda}(\mu)$ for $I^{\lambda}(\mu)$, with the top factor $M_{n} / M_{n-1} \cong M(\mu)$, since $L(\mu)$ is the unique irreducible quotient of $I^{\lambda}(\mu)$. It then follows that $S^{\lambda}(\mu)=M_{n-1}$, and the result is now clear, since $\left(I^{\lambda}(\mu): M(\mu)\right)=(M(\mu): L(\mu))=1$.

Definition 3.10. Let $\Gamma$ be the directed graph whose set of vertices is $\mathfrak{h}^{*}$, with an edge of multiplicity $(M(\nu): L(\mu))$ from $\mu$ to $\nu$ whenever $\mu<\nu$. Define the nonnegative integer $g_{i}(\nu, \mu)$ to be the number of paths of length $i$ in $\Gamma$ from $\mu$ to $\nu$, for all $\mu, \nu \in \mathfrak{h}^{*}$ and all $i \geq 0$, where we take $g_{0}(\nu, \mu)=\delta_{\nu, \mu}$ (Kronecker symbol). More explicitly,

$$
g_{i}(\nu, \mu)=\sum_{\mu=\chi_{0}<\chi_{1}<\cdots<\chi_{1}=\nu}\left(M\left(\chi_{i}\right): L\left(\chi_{i-1}\right)\right) \cdots\left(M\left(\chi_{1}\right): L\left(\chi_{0}\right)\right) .
$$

Remark. By Proposition 3.9, $g_{1}(\nu, \mu)=\left(S^{\lambda}(\nu): M(\mu)\right)$ for any $\mu, \nu \leq \lambda$.

Proposition 3.11. Let $M \in \mathrm{Ob} C(\lambda)$ have a finite VS. Then $M$ has a projective resolution

$$
\cdots \rightarrow P_{n} \stackrel{d_{n}}{\rightarrow} \ldots \stackrel{d_{1}}{\rightarrow} P_{0} \stackrel{d_{0}}{\rightarrow} M \rightarrow 0
$$

in $C(\lambda)$ such that each

$$
P_{n}=\bigoplus_{\nu \leq \lambda}\left[\sum_{\mu \leq \lambda}(M: M(\mu)) g_{n}(\nu, \mu)\right] I^{\lambda}(\nu)
$$

(that is, $P_{n}$ is a finite direct sum of various $I^{\lambda}(\nu)$, and the number of times $I^{\lambda}(\nu)$ occurs as a summand is $\left.\sum_{\mu \leq \lambda}(M: M(\mu)) g_{n}(\nu, \mu)\right)$, and such that each $\operatorname{Im} d_{n}$ has a finite VS with

$$
\left(\operatorname{Im} d_{n}: M(\nu)\right)=\sum_{\mu \leq \lambda}(M: M(\mu)) g_{n}(\nu, \mu) \quad \text { for all } \nu \leq \lambda .
$$

Proof. It suffices, by induction on $n$, to show that there is a short exact sequence

$$
0 \rightarrow S \rightarrow P \rightarrow M \rightarrow 0
$$


such that $P=\bigoplus_{\nu \leq \lambda}(M: M(\nu)) I^{\lambda}(\nu)$, and such that $S$ has a finite VS with $(S: M(\nu))=\sum_{\mu \leq \lambda}(M: M(\mu)) g_{1}(\nu, \mu)$ for any $\nu \leq \lambda$. Let $0=M_{0} \subset M_{1} \subset \cdots \subset M_{k}=M$ be a VS for $M$, with each $M_{i} / M_{i-1} \cong$ $M\left(\mu_{i}\right)$. Now, from the short exact sequences for each $\mu \leq \lambda$

$$
0 \rightarrow S^{\lambda}(\mu) \rightarrow I^{\lambda}(\mu) \rightarrow M(\mu) \rightarrow 0,
$$

it follows by induction on the length $k$ of the VS for $M$ and the Horseshoe Lemma that there is a short exact sequence

$$
0 \rightarrow S \rightarrow P \rightarrow M \rightarrow 0
$$

in which $P=\bigoplus_{i=1}^{k} I^{\lambda}\left(\mu_{i}\right)=\bigoplus_{\nu \leq \lambda}(M: M(\nu)) I^{\lambda}(\nu)$, and in which $S$ has a filtration by submodules $0=S_{0} \subset S_{1} \subset \cdots \subset S_{k}=S$ with each $S_{i} / S_{i-1} \cong S^{\lambda}\left(\mu_{i}\right)$. But then, by Proposition 3.9, we see that $S$ has a finite VS, and for any $\nu \leq \lambda$ we have

$$
\begin{aligned}
(S: M(\nu)) & =\sum_{i=1}^{k}\left(S_{i} / S_{i-1}: M(\nu)\right)=\sum_{i=1}^{k}\left(S^{\lambda}\left(\mu_{i}\right): M(\nu)\right) \\
& =\sum_{i=1}^{k} g_{1}\left(\nu, \mu_{i}\right)=\sum_{\mu \leq \lambda}(M: M(\mu)) g_{1}(\nu, \mu),
\end{aligned}
$$

which was to be shown.

These exact projective resolutions will be useful in our applications, since if $M$ has a finite VS with factors $M\left(\mu_{1}\right), \ldots, M\left(\mu_{k}\right)$ then a projective resolution given by the above proposition is in fact a local projective resolution of $M$ at $\mu$ for any $\mu$ with $\mu \leq \mu_{i}$ for all $i=1, \ldots, k$. Thus, in the case of modules with finite VS, the need for passage to the direct limit is eliminated in the computation of left locally derived functors.

4. Verma series and reverse Verma series. In order to define translation functors from chambers to walls, which will be done in the next section, we need to deal with certain weight modules with infinite dimensional weight spaces and whose set of weights is not bounded above, i.e. modules which are not in any category of the form $C(\lambda)$, or any other category which behaves like what is usually called the category $\mathscr{O}$. The modules which occur may be dealt with by using the idea of reverse Verma series, which were defined in Definition 2.3, and which we study in this section, along with Verma series, defined in Definition 2.2.

We first observe that, by property (iii) of Definition 2.3, one can find a rearrangement $\mu_{1}, \mu_{2}, \ldots$ of the weights $\lambda_{1}, \lambda_{2}, \ldots$ such that $\mu_{i}<\mu_{j}$ 
implies $i<j$. To see this, for each $n=1,2, \ldots$ let $S_{n}=\left\{i \mid \lambda_{i} \leq\right.$ $\left.\lambda_{n}\right\}$, so that $S_{1} \subset S_{2} \subset \ldots$ and $\bigcup_{n>0} S_{n}=\{1,2,3, \ldots\}$. Each $S_{n}$ is finite by property (iii) of the definition, so that we may choose such an arrangement for $S_{1}$, then append weights to obtain such an arrangement for $S_{2}$, etc.

We now prove convenient criteria for determining whether a given weight module has an RVS, or whether it has a VS.

Proposition 4.1. Let $M$ be a weight module. Then $M$ has an RVS if and only if $M$ is a free $U\left(\mathfrak{n}^{-}\right)$-module with a $U\left(\mathfrak{n}^{-}\right)$-basis consisting of weight vectors $\left\{v_{1}, v_{2}, \ldots\right\}$ of weights $\lambda_{1}, \lambda_{2}, \ldots$, respectively, such that for any $\mu \in \mathfrak{h}^{*},\left\{i \mid \lambda_{i} \leq \mu\right\}$ is a finite set.

Proof. If $M$ has an RVS, then we obtain such a $U\left(\mathfrak{n}^{-}\right)$-basis directly from property (iv) of the definition.

Conversely, suppose such $v_{i}$ exist. As in the preceding discussion, we let $\mu_{1}, \mu_{2}, \ldots$ be a rearrangement of $\lambda_{1}, \lambda_{2}, \ldots$ such that $\mu_{i}<\mu_{j}$ implies $i<j$, and let $w_{1}, w_{2}, \ldots$ be the corresponding rearrangement of $v_{1}, v_{2}, \ldots$ Thus $w_{i} \in M_{\mu_{i}}$ for each $i=1,2, \ldots$, and for any $\mu \in$ $\mathfrak{h}^{*},\left\{i \mid \mu_{i} \leq \mu\right\}$ is a finite set. Set $N_{i}=\sum_{j>i} U\left(\mathfrak{n}^{-}\right) w_{j}$. Since $M$ is $U\left(\mathfrak{n}^{-}\right)$-free with basis $\left\{v_{1}, v_{2}, \ldots\right\}=\left\{w_{1}, w_{2}, \ldots\right\}$, it is clear that $M=N_{0} \supset N_{1} \supset \ldots$ and that $\bigcap_{i \geq 0} N_{i}=0$. We now show that each $N_{i}$ is a $\mathfrak{g}$-submodule of $M$. For this, it suffices to show that for any $j=1,2, \ldots, U(\mathfrak{g}) U\left(\mathfrak{n}^{-}\right) w_{j}=U(\mathfrak{g}) w_{j} \subseteq \sum_{k \geq j} U\left(\mathfrak{n}^{-}\right) w_{k}$. Since $U(\mathfrak{g})=U\left(\mathfrak{n}^{-}\right) U(\mathfrak{b})$ by the PBW Theorem, it suffices to show that $U(\mathfrak{b}) w_{j} \subseteq \sum_{k \geq j} U\left(\mathfrak{n}^{-}\right) w_{k}$. But this follows from the ordering of the weights of the weight vectors $w_{j}$ and the fact that $M=\sum_{k>0} U\left(\mathfrak{n}^{-}\right) w_{k}$, when we write $U(\mathfrak{b})=U\left(\mathfrak{n}^{+}\right) U(\mathfrak{h})$ by the PBW Theorem. It remains to show that each $N_{i-1} / N_{i} \cong M\left(\mu_{i}\right)$. Observe that $N_{i-1} / N_{i}$ is generated by $w_{i}+N_{i}$ and is $U\left(\mathfrak{n}^{-}\right)$-free of rank one. By the ordering of the weights $U\left(\mathfrak{n}^{+}\right) w_{i} \subseteq N_{i}$, so that $w_{i}+N_{i}$ is a highest weight vector and $N_{i-1} / N_{i}$ is a highest weight module of weight $\mu_{i}$. Since it is $U\left(\mathfrak{n}^{-}\right)$free, we have $N_{i-1} / N_{i} \cong M\left(\mu_{i}\right)$.

As a corollary of the proof, we obtain the following.

COROLlARY 4.2. If $M$ has an RVS, then it has an RVS $M=M_{0} \supset$ $M_{1} \supset \ldots$ where the order of the factors $M_{i-1} / M_{i} \cong M\left(\lambda_{i}\right)$ satisfies $\lambda_{i}<\lambda_{j}$ implies $i<j$. 
By an entirely analogous argument to that used in Proposition 4.1, we may prove the following proposition and corollary regarding modules with VS.

Proposition 4.3. Let $M$ be a weight module. Then $M$ has a VS if and only if $M$ is a free $U\left(\mathfrak{n}^{-}\right)$-module with a $U\left(\mathfrak{n}^{-}\right)$-basis consisting of weight vectors $\left\{v_{1}, v_{2}, \ldots\right\}$ of weights $\lambda_{1}, \lambda_{2}, \ldots$, respectively, such that for any $\mu \in \mathfrak{h}^{*},\left\{i \mid \lambda_{i} \geq \mu\right\}$ is a finite set.

COROLLARY 4.4. If $M$ has a VS, then it has a VS $0=M_{0} \subset M_{1} \subset \ldots$ where the order of the factors $M_{i} / M_{i-1} \cong M\left(\lambda_{i}\right)$ satisfies $\lambda_{i}>\lambda_{j}$ implies $i<j$.

We now wish to prove certain properties regarding the ordering of factors in an RVS. In particular, we wish to be able to order the factors in such a way that all the Verma factors whose highest weights lie in a certain set (which we will later take to be a Weyl group orbit or a union of such orbits) may be assumed to occur as consecutive factors in the RVS. The main results are Theorems 4.14 and 4.19. We begin with some properties of Hom and Ext.

Proposition 4.5 [10, Proposition 2.6]. Let $\mu, \nu \in \mathfrak{h}^{*}$. Then $(M(\nu)$ : $L(\mu)) \neq 0$ if and only if $\operatorname{Hom}(M(\mu), M(\nu)) \neq 0$.

Proposition 4.6. Let $\lambda, \mu, \nu \in \mathfrak{h}^{*}$ such that $\mu, \nu \leq \lambda$, and let $n \geq 0$. If $M$ is a highest weight module of weight $\nu$ and $\operatorname{Ext}_{C(\lambda)}^{n}(M(\mu), M) \neq$ 0 , then there exist weights $\mu=\chi_{n}<\chi_{n-1}<\cdots<\chi_{0} \leq \nu$ such that $\operatorname{Hom}\left(M\left(\chi_{i}\right), M\left(\chi_{i-1}\right)\right) \neq 0$ for all $i=1, \ldots, n$ and such that $\operatorname{Hom}\left(M\left(\chi_{0}\right), M(\nu)\right) \neq 0$.

Proof. We use induction on $n$. For $n=0$, if $\operatorname{Hom}(M(\mu), M) \neq 0$ then $L(\mu)$ is a composition factor of $M$, and hence $(M(\nu): L(\mu)) \neq 0$ since $M$ has highest weight $\nu$. But then, by Proposition 4.5, we have $\operatorname{Hom}(M(\mu), M(\nu)) \neq 0$, and we are done by taking $\chi_{0}=\mu$.

For $n>0$, use the short exact sequence

$$
0 \rightarrow S^{\lambda}(\mu) \rightarrow I^{\lambda}(\mu) \rightarrow M(\mu) \rightarrow 0
$$

and the projectivity of $I^{\lambda}(\mu)$ to conclude that if $\operatorname{Ext}_{C(\lambda)}^{n}(M(\mu), M)$ is nonzero, then so is $\operatorname{Ext}_{C(\lambda)}^{n-1}\left(S^{\lambda}(\mu), M\right)$. But then, by Proposition 3.9, we have $\operatorname{Ext}_{C(\lambda)}^{n-1}(M(\chi), M) \neq 0$ for some Verma factor $M(\chi)$ in the VS for $S^{\lambda}(\mu)$, and this Verma factor satisfies both $\chi>\mu$ and 
$(M(\chi): L(\mu)) \neq 0$. Thus by Proposition 4.5, $\operatorname{Hom}(M(\mu), M(\chi)) \neq 0$, so that we are done by applying the inductive hypothesis to $M(\chi)$.

COROllary 4.7. Let $\mu, \nu \in \mathfrak{h}^{*}$, and let $M$ be a highest weight module of weight $\nu$. If $\operatorname{Ext}(M(\mu), M) \neq 0$, then $\nu>\mu$, and $\operatorname{Hom}(M(\mu), M(\nu))$ $\neq 0$.

Proof. If we have a nonsplit short exact sequence

$$
0 \rightarrow M \rightarrow N \rightarrow M(\mu) \rightarrow 0
$$

in the category of weight modules, then we must have $\nu>\mu$, since otherwise the universal property of $M(\mu)$ would give a splitting map. Thus, taking $\lambda=\nu$, this is a nonsplit sequence in $C(\lambda)$, so that $\operatorname{Ext}_{C(\lambda)}^{1}(M(\mu), M) \neq 0$. By the above proposition, we obtain a chain of imbeddings $M(\mu)=M\left(\chi_{1}\right) \subset M\left(\chi_{0}\right) \subseteq M(\nu)$.

LEMMA 4.8. Let $M=M_{0} \supset M_{1} \supset \cdots$ be an RVS for the weight module $M$ with factors $M_{i-1} / M_{i} \cong M\left(\lambda_{i}\right)$ and weight vectors $v_{i} \in M_{\lambda_{i}}$ as in the definition. Suppose that for some $n, \operatorname{Ext}\left(M\left(\lambda_{n}\right), M\left(\lambda_{n+1}\right)\right)=$ 0 . Then there is a submodule $M_{n}^{\prime}$ of $M_{n-1}$ such that $M=M_{0} \supset$ $M_{1} \supset \cdots \supset M_{n-1} \supset M_{n}^{\prime} \supset M_{n+1} \supset \cdots$ is also an RVS for $M$ with $M_{n-1} / M_{n}^{\prime} \cong M\left(\lambda_{n+1}\right)$ and $M_{n}^{\prime} / M_{n+1} \cong M\left(\lambda_{n}\right)$.

Proof. By hypothesis, the short exact sequence

$$
0 \rightarrow M_{n} / M_{n+1} \rightarrow M_{n-1} / M_{n+1} \rightarrow M_{n-1} / M_{n} \rightarrow 0
$$

splits, so that there is a submodule $M_{n}^{\prime}$ of $M_{n-1}$ such that we have an internal direct sum $M_{n-1} / M_{n+1}=M_{n} / M_{n+1} \oplus M_{n}^{\prime} / M_{n+1}$ with $M_{n}^{\prime} / M_{n+1}=M_{n-1} / M_{n} \cong M\left(\lambda_{n}\right)$. From this it is clear that $M_{n-1} / M_{n}^{\prime}=$ $M_{n} / M_{n+1} \cong M\left(\lambda_{n+1}\right)$. For $i \neq n, n+1$, let $w_{i}=v_{i}$. Choose $w_{n+1} \in$ $M_{\lambda_{n}}$ such that $w_{n+1}+M_{n+1}$ is a highest weight vector for $M_{n}^{\prime} / M_{n+1}$, and set $w_{n}=v_{n+1}$. Then the weight vectors $w_{1}, w_{2}, \ldots, w_{n}, w_{n+1}, \ldots$ satisfy the hypotheses of the definition for the submodule sequence $M=M_{0} \supset M_{1} \supset \cdots \supset M_{n-1} \supset M_{n}^{\prime} \supset M_{n+1} \supset \cdots$ to be an RVS for $M$.

Proposition 4.9. Let $M=M_{0} \supset M_{1} \supset \cdots$ be an $R V S$ for $M$ with factors $F_{i}=M_{i-1} / M_{i} \cong M\left(\lambda_{i}\right)$. Let $S \subseteq \mathfrak{h}^{*}$ be a set of weights such that $\lambda_{i} \in S$ for all $i$, and suppose $S=S_{1} \cup S_{2}$ is a disjoint union such that $\operatorname{Hom}(M(\mu), M(\nu))=0$ whenever $\mu \in S_{1}$ and $\nu \in S_{2}$. Further suppose that $\left\{i \mid \lambda_{i} \in S_{2}\right\}$ is a finite set. Then $M$ has a submodule $N$ such that $N$ 
has an RVS whose factors are those $F_{i}$ with $\lambda_{i} \in S_{1}$ and such that $M / N$ has a finite RVS (or finite VS) whose factors are those $F_{i}$ with $\lambda_{i} \in S_{2}$.

Proof. Observe that $\operatorname{Ext}(M(\mu), M(\nu))=0$ whenever $\mu \in S_{1}$ and $\nu \in S_{2}$ by Corollary 4.7. Thus, we may apply the lemma a finite number of times, switching the order of the factors, to obtain an RVS $M=M_{0}^{\prime} \supset M_{1}^{\prime} \supset \cdots \supset M_{n}^{\prime} \supset \cdots$ for $M$, where for $i=1, \ldots, n$ we have $M_{i-1}^{\prime} / M_{i}^{\prime} \cong M\left(\mu_{i}\right)$ with $\mu_{i} \in S_{2}$ and for $i>n$ we have $M_{i-1}^{\prime} / M_{i}^{\prime} \cong M\left(\mu_{i}\right)$ with $\mu_{i} \in S_{1}$, and where the sequence $\mu_{1}, \mu_{2}, \ldots$ is a rearrangement of $\lambda_{1}, \lambda_{2}, \ldots$ Setting $N=M_{n}^{\prime}$ gives the result.

Proposition 4.10. Let $M=M_{0} \supset M_{1} \supset \cdots$ and $M^{\prime}=M_{0}^{\prime} \supset$ $M_{1}^{\prime} \supset \cdots$ each satisfy the hypotheses of Proposition 4.9 for the same set of weights $S=S_{1} \cup S_{2}$, and let $N \subseteq M$ and $N^{\prime} \subseteq M^{\prime}$ be as in the conclusion of the proposition. If $\phi \in \operatorname{Hom}\left(M, M^{\prime}\right)$, then $\phi(N) \subseteq N^{\prime}$, so that $\phi$ induces maps $\phi_{1}: N \rightarrow N^{\prime}$ and $\phi_{2}: M / N \rightarrow M^{\prime} / N^{\prime}$.

Proof. If this were not the case, then we would have a nonzero composition of $\mathfrak{g}$-module maps

$$
N \stackrel{\phi}{\rightarrow} M^{\prime} \stackrel{\pi}{\rightarrow} M^{\prime} / N^{\prime}
$$

where $\pi$ is the canonical epimorphism. Let $N=N_{0} \supset N_{1} \supset \cdots$ be an RVS for $N$, with $N_{i-1} / N_{i} \cong M\left(\mu_{i}\right)$ for some $\mu_{i} \in S_{1}$ for each $i$, and let $M^{\prime} / N^{\prime}=V_{0} \supset V_{1} \supset \cdots \supset V_{n}=0$ be a finite RVS for $M^{\prime} / N^{\prime}$, with $V_{j-1} / V_{j} \cong M\left(\nu_{j}\right)$ for some $\nu_{j} \in S_{2}$ for each $j$. Since for each $j,\left\{i \mid \mu_{i} \leq \nu_{j}\right\}$ is a finite set, there is some $k$ such that $0=$ $\left(M^{\prime} / N^{\prime}\right)_{\mu_{k+1}}=\left(M^{\prime} / N^{\prime}\right)_{\mu_{k+2}}=\cdots$. Choosing weight vectors $v_{j} \in N_{\mu_{j}}$ as in the definition of an RVS, since $N_{k}=\sum_{j>k} U\left(\mathfrak{n}^{-}\right) v_{j}$ and $\pi \circ$ $\phi\left(v_{j}\right)=0$ for all $j>k$, we see that $\pi \circ \phi\left(N_{k}\right)=0$. This implies that $\operatorname{Hom}\left(N / N_{k}, M^{\prime} / N^{\prime}\right) \neq 0$. But each of the modules $N / N_{k}$ and $M^{\prime} / N^{\prime}$ has a finite RVS, so this implies that $\operatorname{Hom}\left(M\left(\mu_{i}\right), M\left(\nu_{j}\right)\right) \neq 0$ for some $i$ and $j$. Since $\mu_{i} \in S_{1}$ and $\nu_{j} \in S_{2}$, we have a contradiction to the hypothesis on $S_{1}$ and $S_{2}$. Thus $\phi(N) \subseteq N^{\prime}$.

Corollary 4.11. Let $M$ and $S=S_{1} \cup S_{2}$ satisfy the hypotheses of Proposition 4.9. Then the submodule $N$ given in the conclusion of that proposition is unique (that is, it does not depend on the choice of RVS for $M)$.

Proof. Apply Proposition 4.10 to the case where $\phi: M \rightarrow M$ is the identity map. 
Proposition 4.12. Let $M=M_{0} \supset M_{1} \supset \cdots$ and $M=M_{0}^{\prime} \supset M_{1}^{\prime} \supset$ ‥ be two RVS for $M$ with $M_{i-1} / M_{i} \cong M\left(\lambda_{i}\right)$ and $M_{i-1}^{\prime} / M_{i}^{\prime} \cong M\left(\mu_{i}\right)$ for $i=1,2, \cdots$, and let $\nu \in \mathfrak{h}^{*}$. Then $\left|\left\{i \mid \lambda_{i}=\nu\right\}\right|=\left|\left\{i \mid \mu_{i}=\nu\right\}\right|$.

Proof. Let $S=\left\{\lambda_{i} \mid i \geq 1\right\} \cup\left\{\mu_{i} \mid i \geq 1\right\}, S_{1}=S \cap\{\chi \mid \chi \not \nu\}$, and $S_{2}=S \cap\{\chi \mid \chi \leq \nu\}$. Observe that $S=S_{1} \cup S_{2}$ is a disjoint union and $\operatorname{Hom}(M(\mu), M(\nu))=0$ whenever $\mu \in S_{1}$ and $\nu \in S_{2}$. Also, $\left\{i \mid \lambda_{i} \in S_{2}\right\}$ and $\left\{i \mid \mu_{i} \in S_{2}\right\}$ are finite sets, by the definition of RVS. Thus, we may apply Proposition 4.9 to obtain submodules $N$ and $N^{\prime}$ of $M$ such that $M / N$ (respectively, $M / N^{\prime}$ ) has a finite RVS whose factors are those $M\left(\lambda_{i}\right)$ (respectively, $M\left(\mu_{i}\right)$ ) such that $\lambda_{i} \in S_{2}$ (respectively, $\mu_{i} \in S_{2}$ ). But by the uniqueness asserted in Corollary 4.11, $N=N^{\prime}$, so that

$$
\left|\left\{i \mid \lambda_{i}=\nu\right\}\right|=(M / N: M(\nu))=\left(M / N^{\prime}: M(\nu)\right)=\left|\left\{i \mid \mu_{i}=\nu\right\}\right| .
$$

REMARK. In general, modules with RVS have infinite dimensional weight spaces, so that the above proposition could not be proved by character considerations, as is done in the case of VS. In fact, we have used the result for modules with VS by reducing the RVS to a finite RVS, which is a VS.

Proposition 4.12 allows us to make the following definition.

Definition 4.13. If $M=M_{0} \supset M_{1} \supset \cdots$ is an RVS with factors $M_{i-1} / M_{i} \cong M\left(\lambda_{1}\right)$ for $i=1,2, \ldots$ and $\nu \in \mathfrak{h}^{*}$, then we define $(M: M(\nu))=\left|\left\{i \mid \lambda_{i}=\nu\right\}\right|$.

THEOREM 4.14. Let $S \subseteq \mathfrak{h}^{*}$ be a set of weights, and suppose we are given a finite disjoint union $S=S_{1} \cup \cdots \cup S_{n}$ such that $\operatorname{Hom}(M(\mu), M(\nu))$ $=0$ whenever $\mu \in S_{i}$ and $\nu \in S_{j}$ with $i<j$. Then the following hold.

(i) If $M=M_{0} \supset M_{1} \supset \cdots$ is an RVS for $M$ with factors $F_{i}=$ $M_{i-1} / M_{i} \cong M\left(\lambda_{i}\right)$ such that $\lambda_{i} \in S$ for all $i$, and if $\left\{i \mid \lambda_{i} \in S_{k}\right\}$ is a finite set for each $k=2, \ldots, n$, then there are unique submodules $M=$ $N_{n} \supset N_{n-1} \supset \cdots \supset N_{0}=0$ such that each $N_{k} / N_{k-1}$ for $k=2, \ldots, n$ has a finite RVS whose factors are those $F_{i}$ with $\lambda_{i} \in S_{k}$, and such that $N_{1} / N_{0}$ has an RVS whose factors are those $F_{i}$ with $\lambda_{i} \in S_{1}$.

(ii) If $M=N_{n} \supset N_{n-1} \supset \cdots \supset N_{0}=0$ and $M^{\prime}=N_{n}^{\prime} \supset N_{n-1}^{\prime} \supset \cdots \supset$ $N_{0}^{\prime}=0$ both are filtrations by submodules satisfying the conclusion of (i) above, then any $\phi \in \operatorname{Hom}\left(M, M^{\prime}\right)$ satisfies $\phi\left(N_{k}\right) \subseteq N_{k}^{\prime}$ for all $k=0,1, \ldots, n$, so that $\phi$ induces maps $\phi_{k}: N_{k} / N_{k-1} \rightarrow N_{k}^{\prime} / N_{k-1}^{\prime}$ for $k=1, \ldots, n$.

Proof. This follows, by induction on $n$, from Propositions 4.9 and 4.10 and Corollary 4.11 , when we write $S=\left(S_{1} \cup \cdots \cup S_{n-1}\right) \cup S_{n}$. 
Using analogous arguments, similar results (without the finiteness hypotheses) were established in [11]. We state them here for later use.

Proposition 4.15 [11, Corollaries 4.4 and 4.6 and Proposition 4.5]. Let $0=M_{0} \subset M_{1} \subset \cdots$ be a VS for $M$ with factors $F_{l}=M_{i} / M_{i-1} \cong$ $M\left(\lambda_{i}\right)$ for each $i$, and let $S \subseteq \mathfrak{h}^{*}$ be a set of weights such that $\lambda_{i} \in S$ for all $i$. Suppose $S=S_{1} \cup \cdots \cup S_{n}$ is a finite disjoint union such that $\operatorname{Hom}(M(\mu), M(\nu))=0$ whenever $\mu \in S_{i}$ and $\nu \in S_{j}$ with $i<j$. Then there are unique submodules $0=N_{0} \subset N_{1} \subset \cdots \subset N_{n}=M$ such that each $N_{k} / N_{k-1}$ for $k=1, \ldots, n$ has a VS whose factors are those $F_{i}$ with $\lambda_{i} \in S_{k}$.

Also, if $0=M_{0}^{\prime} \subset M_{1}^{\prime} \subset \cdots$ is a VS for $M^{\prime}$ satisfying the same hypotheses, and if $0=N_{0}^{\prime} \subset N_{1}^{\prime} \subset \cdots \subset N_{n}^{\prime}=M^{\prime}$ is its filtration as stated in the first paragraph, then any $\phi \in \operatorname{Hom}\left(M, M^{\prime}\right)$ satisfies $\phi\left(N_{k}\right) \subseteq N_{k}^{\prime}$ for all $k=0,1, \ldots, n$, so that $\phi$ induces maps $\phi_{k}: N_{k} / N_{k-1} \rightarrow N_{k}^{\prime} / N_{k-1}^{\prime}$ for $k=1, \ldots, n$.

We now turn to certain situations in which modules can be shown to have RVS or VS.

Proposition 4.16. Suppose that $M$ has an RVS and that $N$ is a lowest weight module with lowest weight $\mu$. Then $M \otimes_{K} N$ has an RVS, and $\left(M \otimes_{K} N: M(\nu)\right)=\sum_{\chi \geq \mu}(M: M(\nu-\chi)) \operatorname{dim} N_{\chi}$ for any $\nu \in \mathfrak{h}^{*}$.

Proof. Choose a free $U\left(\mathfrak{n}^{-}\right)$-basis $\left\{v_{1}, v_{2}, \ldots\right\}$ for $M$ consisting of weight vectors, as in Proposition 4.1. Taking a $K$-basis $\left\{w_{1}, w_{2}, \ldots\right\}$ of weight vectors for $N$, we see that $M \otimes_{K} N$ is a free $U\left(\mathfrak{n}^{-}\right)$-module with basis $\left\{v_{i} \otimes w_{j} \mid i, j \geq 1\right\}$. Since $N$ is a lowest weight module, it is clear that the finiteness hypothesis of Proposition 4.1 is satisfied, which implies that $M \otimes_{K} N$ has an RVS. By the proof of Proposition 4.1, $\left(M \otimes_{K} N: M(\nu)\right)$ is the number of $v_{i} \otimes w_{j}$ which have weight $\nu$, and this is clearly $\sum_{\chi \geq \mu}(M: M(\nu-\chi)) \operatorname{dim} N_{\chi}$.

REMARK. This shows that, in particular, $M(\lambda) \otimes_{K} \Gamma(\mu)$ has an RVS for any $\lambda, \mu \in \mathfrak{h}^{*}$.

Entirely analogous arguments, using Proposition 4.3 in place of Proposition 4.1, may be used to show the following result.

Proposition 4.17. Suppose that $M$ has a VS and that $N$ is a highest weight module with highest weight $\mu$. Then $M \otimes_{K} N$ has a VS, and $\left(M \otimes_{K} N: M(\nu)\right)=\sum_{\chi \leq \mu}(M: M(\nu-\chi)) \operatorname{dim} N_{\chi}$ for any $\nu \in \mathfrak{h}^{*}$. 
We close this section by investigating exactness properties of the maps involved in the situation of Theorem 4.14 and Proposition 4.15.

LemMa 4.18. Suppose that

$$
0 \rightarrow M^{\prime} \stackrel{\phi}{\rightarrow} M \stackrel{\psi}{\rightarrow} M^{\prime \prime} \rightarrow 0
$$

is a short exact sequence of $\mathfrak{g}$-modules with RVS, each of which satisfies the hypotheses of Proposition 4.9 together with the set of weights $S=$ $S_{1} \cup S_{2}$. Let $N^{\prime} \subseteq M^{\prime}, N \subseteq M$, and $N^{\prime \prime} \subseteq M^{\prime \prime}$ be as stated in the conclusion of Proposition 4.9. Then the sequence of induced maps given by Proposition 4.10

$$
0 \rightarrow M^{\prime} / N^{\prime} \stackrel{\phi_{2}}{\rightarrow} M / N \stackrel{\psi_{2}}{\rightarrow} M^{\prime \prime} / N^{\prime \prime} \rightarrow 0
$$

is also exact.

Proof. We first show that the induced map $\psi_{1}: N \rightarrow N^{\prime \prime}$ is an epimorphism. Let $v_{1}^{\prime \prime}, v_{2}^{\prime \prime}, \ldots$ be canonical generators for $N^{\prime \prime}$ as in the definition of RVS, of weights $\lambda_{1}, \lambda_{2}, \ldots$, respectively, so that each $\lambda_{i} \in S_{1}$. Observe that since $M / N$ has a finite RVS, for some $n$ we must have $M_{\lambda_{1}}=N_{\lambda_{1}}$ for all $i>n$. But $\psi$ is surjective, so this shows that for all $i>n, v_{i}^{\prime \prime} \in \psi\left(M_{\lambda_{1}}\right)=\psi_{1}\left(N_{\lambda_{1}}\right)$. If we can show that $v_{i}^{\prime \prime} \in \operatorname{Im} \psi_{1}$ for all $i \geq 1$, we will have proved our assertion regarding $\psi_{1}$. Suppose that this is not the case. Then by the preceding argument, we see that there must be some $v_{i}^{\prime \prime} \notin \operatorname{Im} \psi_{1}$ such that $v_{j}^{\prime \prime} \in \operatorname{Im} \psi_{1}$ for all $j>i$. Now $\mathfrak{n}^{+} \cdot v_{i}^{\prime \prime} \subseteq \sum_{j>i} U\left(\mathfrak{n}^{-}\right) v_{j}^{\prime \prime} \subseteq \operatorname{Im} \psi_{1}$, so that $v_{i}^{\prime \prime}$ is a maximal vector in $N^{\prime \prime} / \operatorname{Im} \psi_{1}$ of weight $\lambda_{i}$. Thus $L\left(\lambda_{i}\right)$ is a composition factor of $N^{\prime \prime} / \operatorname{Im} \psi_{1}$, and hence $L\left(\lambda_{i}\right)$ is also a composition factor of $M^{\prime \prime} / \operatorname{Im} \psi_{1}$. But we have an epimorphism $M / N \rightarrow M^{\prime \prime} / \operatorname{Im} \psi_{1}$ induced by $\psi$, so this implies that $\left(M / N: L\left(\lambda_{i}\right)\right) \neq 0$. Since $M / N$ has a finite RVS whose factors are of the form $M(\mu)$ with $\mu \in S_{2}$, we have that $\left(M(\mu): L\left(\lambda_{i}\right)\right) \neq 0$ for some $\mu \in S_{2}$, and hence $\operatorname{Hom}\left(M\left(\lambda_{i}\right), M(\mu)\right) \neq 0$ by Proposition 4.5. This contradicts the hypothesis on $S_{1}$ and $S_{2}$, since $\lambda_{i} \in S_{1}$ and $\mu \in S_{2}$. Thus $\psi_{1}$ is an epimorphism.

Now $\psi_{2} \circ \phi_{2}=0$, since it is induced by $\psi \circ \phi$, so that $\operatorname{Im} \phi_{2} \subseteq \operatorname{Ker} \psi_{2}$. Also $\psi_{2}$ is surjective, since it is induced by $\psi$. Suppose $x+N \in \operatorname{Ker} \psi_{2}$. Then $\psi_{2}(x+N)=\psi(x)+N^{\prime \prime}=N^{\prime \prime}$, so we have $\psi(x) \in N^{\prime \prime}$. By the above paragraph, there exists $y \in N$ with $\psi(x)=\psi(y)$. Thus $x-y \in \operatorname{Ker} \psi=\operatorname{Im} \phi$, and there is some $z \in M^{\prime}$ with $\phi(z)=x-y$. But then $\phi_{2}\left(z+N^{\prime}\right)=\phi(z)+N=x-y+N=x+N$. Therefore $\operatorname{Ker} \psi_{2}=\operatorname{Im} \phi_{2}$. 
It remains to show that $\operatorname{Ker} \phi_{2}=0$. By what we have already shown, we have $\operatorname{ch}(M / N)=\operatorname{ch}\left(M^{\prime} / N^{\prime}\right)+\operatorname{ch}\left(M^{\prime \prime} / N^{\prime \prime}\right)+\operatorname{ch}\left(\operatorname{Ker} \phi_{2}\right)$. On the other hand, each of $M / N, M^{\prime} / N^{\prime}$, and $M^{\prime \prime} / N^{\prime \prime}$ has a finite RVS and for any $\nu \in S_{2}$,

$$
\begin{aligned}
(M / N: M(\nu)) & =(M: M(\nu))=\left(M^{\prime}: M(\nu)\right)+\left(M^{\prime \prime}: M(\nu)\right) \\
& =\left(M^{\prime} / N^{\prime}: M(\nu)\right)+\left(M^{\prime \prime} / N^{\prime \prime}: M(\nu)\right),
\end{aligned}
$$

and hence $\operatorname{ch}(M / N)=\operatorname{ch}\left(M^{\prime} / N^{\prime}\right)+\operatorname{ch}\left(M^{\prime \prime} / N^{\prime \prime}\right)$. Thus $\operatorname{ch}\left(\operatorname{Ker} \phi_{2}\right)=0$, which implies $\operatorname{Ker} \phi_{2}=0$.

THEOREM 4.19. Let

$$
0 \rightarrow M^{\prime} \stackrel{\phi}{\rightarrow} M \stackrel{\psi}{\rightarrow} M^{\prime \prime} \rightarrow 0
$$

be an exact sequence of $\mathfrak{g}$-modules, each of which satisfies the hypotheses of Theorem 4.14 together with the set of weights $S=S_{1} \cup \cdots \cup S_{n}$. Let $M^{\prime}=N_{n}^{\prime} \supset N_{n-1}^{\prime} \supset \cdots \supset N_{0}^{\prime}=0$, let $M=N_{n} \supset N_{n-1} \supset \cdots \supset N_{0}=0$, and let $M^{\prime \prime}=N_{n}^{\prime \prime} \supset N_{n-1}^{\prime \prime} \supset \cdots \supset N_{0}^{\prime \prime}=0$ be the filtrations given by the conclusion of Theorem 4.14. Then for each $k=2, \ldots, n$ the induced sequence

$$
0 \rightarrow N_{k}^{\prime} / N_{k-1}^{\prime} \stackrel{\phi_{k}}{\rightarrow} N_{k} / N_{k-1} \stackrel{\psi_{k}}{\rightarrow} N_{k}^{\prime \prime} / N_{k-1}^{\prime \prime} \rightarrow 0
$$

is exact.

Proof. Writing $S=S_{1} \cup\left(S_{2} \cup \cdots \cup S_{n}\right)$, the lemma reduces us to the case of finite VS, which was proved in [11, Theorem 4.8] with somewhat weaker hypotheses.

For completeness, we include the following result on modules with VS, which is the analogue of Theorem 4.19.

Proposition 4.20 [11, Theorem 4.8]. Let

$$
0 \rightarrow M^{\prime} \stackrel{\phi}{\rightarrow} M \stackrel{\psi}{\rightarrow} M^{\prime \prime} \rightarrow 0
$$

be an exact sequence of $\mathfrak{g}$-modules, each of which satisfies the hypothesis of Proposition 4.15 together with the set of weights $S=S_{1} \cup \cdots \cup S_{n}$. Let $0=N_{0}^{\prime} \subset \cdots \subset N_{n}^{\prime}=M^{\prime}$, let $0=N_{0} \subset \cdots \subset N_{n}=M$, and let $0=N_{0}^{\prime \prime} \subset \cdots \subset N_{n}^{\prime \prime}=M^{\prime \prime}$ be the filtrations given by the conclusion of Proposition 4.15. Then for each $k=1, \ldots, n$ the induced sequence

$$
0 \rightarrow N_{k}^{\prime} / N_{k-1}^{\prime} \stackrel{\phi_{k}}{\rightarrow} N_{k} / N_{k-1} \stackrel{\psi_{k}}{\rightarrow} N_{k}^{\prime \prime} / N_{k-1}^{\prime \prime} \rightarrow 0
$$

is exact. 
5. W-orbits and translation functors. We now apply the results of the previous section on VS and RVS to define translation functors. The basic idea is to "translate" Verma modules from one Weyl group orbit in $\mathfrak{h}^{*}$ (under the dot action of $W$ ) to another orbit. When we observe that certain unions of Weyl group orbits satisfy the hypotheses of the results of $\S 4$, those results may be applied to modules with VS or with RVS in this situation. Since the projectives have finite VS (or RVS), we may then apply the results of $\S 3$ to define translation functors for modules which do not have VS or RVS.

Throughout this section, fix $\lambda^{\prime}, \lambda^{\prime \prime} \in \mathfrak{h}^{*}$ such that $\lambda^{\prime}+\rho \in P^{+}, \lambda^{\prime \prime}+\rho \in$ $P^{+}$, and $\lambda^{\prime}-\lambda^{\prime \prime} \in P^{+}$. Thus, with respect to the dot action of $W$, on $\mathfrak{h}^{*}$, both $\lambda^{\prime}$ and $\lambda^{\prime \prime}$ are in the closure of the dominant chamber, and $\lambda^{\prime \prime}$ lies in the closure of the facette containing $\lambda^{\prime}$. We will translate back and forth between the orbits $W \cdot \lambda^{\prime}$ and $W \cdot \lambda^{\prime \prime}$ using the modules $L\left(\lambda^{\prime}-\lambda^{\prime \prime}\right)$ and $\Gamma\left(\lambda^{\prime \prime}-\lambda^{\prime}\right)$. Note that since $\lambda^{\prime}-\lambda^{\prime \prime} \in P^{+}$we have that both $L\left(\lambda^{\prime}-\lambda^{\prime \prime}\right)$ and $\Gamma\left(\lambda^{\prime \prime}-\lambda^{\prime}\right)$ are integrable modules (that is, they are direct sums of finite dimensional $\mathfrak{a}_{i}$-modules for any $\left.i=1, \ldots, l\right)$, so that an easy application of the representation theory of $\operatorname{sl}(2, K)$ and induction on $l(w)$ gives $\operatorname{dim} L\left(\lambda^{\prime}-\lambda^{\prime \prime}\right)_{w \mu}=\operatorname{dim} L\left(\lambda^{\prime}-\lambda^{\prime \prime}\right)_{\mu}$ and $\operatorname{dim} \Gamma\left(\lambda^{\prime \prime}-\lambda^{\prime}\right)_{w \mu}=\operatorname{dim} \Gamma\left(\lambda^{\prime \prime}-\lambda^{\prime}\right)_{\mu}$ for all $w \in W$ and all $\mu \in \mathfrak{h}^{*}$.

We further assume that the stabilizer $W_{\lambda^{\prime}}$, of $\lambda^{\prime}$ in $W$ under the dot action has finite index in $W_{\lambda^{\prime \prime}}$ (cf. Remark after Corollary 5.10).

Proposition 5.1 [11, Lemma 5.5]. Let $\nu \in \mathfrak{h}^{*}$ such that $\nu+\rho \in P^{+}$, and set $S_{1}=\left\{\mu \in \mathfrak{h}^{*} \mid w \cdot \mu \not \leq \nu\right.$ for some $\left.w \in W\right\}, S_{2}=W \cdot \nu$, and $S_{3}=\left\{\mu \in \mathfrak{h}^{*} \mid w \cdot \mu<\nu\right.$ for all $\left.w \in W\right\}$. Then $\operatorname{Hom}(M(\lambda), M(\mu))=0$ whenever $\lambda \in S_{i}$ and $\mu \in S_{j}$ with $i<j$.

In light of this proposition, we may use our results on VS and RVS to make the following definition.

Definition 5.2. Let $\nu \in \mathfrak{h}^{*}$ such that $\nu+\rho \in P^{+}$, and let $S_{1}, S_{2}$, and $S_{3}$ be as specified in Proposition 5.1. If $M$ has an RVS satisfying the hypotheses of Theorem 4.14, together with the disjoint union $S_{1} \cup$ $S_{2} \cup S_{3}$, and if $M=N_{3} \supset N_{2} \supset N_{1} \supset N_{0}=0$ is the filtration of submodules given by Theorem 4.15 , then we denote the subquotient $N_{2} / N_{1}$ by $M^{(\nu)}$, so that $M^{(\nu)}$ has a finite VS all of whose factors are of the form $M(w \cdot \nu)$ for some $w \in W$. Furthermore, if $M^{\prime}=N_{3}^{\prime} \supset$ $N_{2}^{\prime} \supset N_{1}^{\prime} \supset N_{0}^{\prime}=0$ is another such module with filtration, and if $\phi \in$ $\operatorname{Hom}\left(M, M^{\prime}\right)$, then we denote the induced map $\phi_{2}: N_{2} / N_{1} \rightarrow N_{2}^{\prime} / N_{1}^{\prime}$ by $\phi^{(\nu)}: M^{(\nu)} \rightarrow M^{(\nu)}$. 
We define modules $M^{(\nu)}$ and maps $\phi^{(\nu)}$ in an analogous manner for modules with VS, using Proposition 4.15 in place of Theorem 4.14.

REMARK. Although we use the same notation in both cases, this should not lead to any confusion, since it will be clear from the context whether we are dealing with modules with VS or RVS, and in either case $M^{(\nu)}$ is simply the subquotient with those Verma factors of the form $M(w \cdot \nu)$ which occur in the original VS or RVS, and $\phi^{(\nu)}$ is simply the map induced by $\phi$ on the subquotients.

We are now ready to define the translation functors between the orbits $W \cdot \lambda^{\prime}$ and $W \cdot \lambda^{\prime \prime}$.

Definition 5.3. Let $F$ be the functor from the full subcategory of $C\left(\lambda^{\prime \prime}\right)$ whose objects are finite direct sums of various $I^{\lambda^{\prime \prime}}(\nu)$ to the category of weight modules defined by $F M=\left(M^{\left(\lambda^{\prime \prime}\right)} \otimes_{K} L\left(\lambda^{\prime}-\lambda^{\prime \prime}\right)\right)^{\left(\lambda^{\prime}\right)}$ and $F \phi=\left(\phi^{\left(\lambda^{\prime \prime}\right)} \otimes 1\right)^{\left(\lambda^{\prime}\right)}$. Then the $q$ th translation functor $T_{\lambda^{\prime \prime}, q}^{\lambda^{\prime}}(q=$ $0,1, \ldots)$ from $C\left(\lambda^{\prime \prime}\right)$ to the category of weight modules is defined by $T_{\lambda^{\prime \prime}, q}^{\lambda^{\prime}}=L_{q} F$, that is, the $q$ th left derived functor of $F$ as defined in Definition 3.6.

Similarly, letting $G$ be the functor from the full subcategory of $C\left(\lambda^{\prime}\right)$ whose objects are finite direct sums of various $I^{\lambda^{\prime}}(\nu)$ to the category of weight modules defined by $G M=\left(M^{\left(\lambda^{\prime}\right)} \otimes_{K} \Gamma\left(\lambda^{\prime \prime}-\lambda^{\prime}\right)\right)^{\left(\lambda^{\prime \prime}\right)}$ and $G \phi=\left(\phi^{\left(\lambda^{\prime}\right)} \otimes 1\right)^{\left(\lambda^{\prime \prime}\right)}$, we define the $q$ th translation functor $T_{\lambda^{\prime}, q}^{\lambda^{\prime \prime}}=L_{q} G$. We set $T_{\lambda^{\prime \prime}}^{\lambda^{\prime}}=T_{\lambda^{\prime \prime}, 0}^{\lambda^{\prime}}$ and $T_{\lambda^{\prime}}^{\lambda^{\prime \prime}}=T_{\lambda^{\prime}, 0}^{\lambda^{\prime \prime}}$.

REMARK. For any particular $\mu \in \mathfrak{h}^{*}$, and any $M \in \mathrm{Ob} C\left(\lambda^{\prime \prime}\right)$, $\left(L_{q, \mu} F\right) M \in \mathrm{Ob} C\left(\lambda^{\prime}\right)$ for each $q=0,1, \ldots$, but when taking the direct limit to obtain $T_{\lambda^{\prime \prime}, q}^{\lambda^{\prime}} M$ we might not remain in the category $C\left(\lambda^{\prime}\right)$, since the weight spaces may no longer be finite dimensional. A similar remark applies to $T_{\lambda^{\prime}, q}^{\lambda^{\prime \prime}}$. One of the results of this section will be that $T_{\lambda^{\prime \prime}, q}^{\lambda^{\prime}} M \in \mathrm{Ob} C\left(\lambda^{\prime}\right)$, so that since $C\left(\lambda^{\prime}\right)$ is a full subcategory of the category of weight modules, this result will allow us to view $T_{\lambda^{\prime \prime}, q}^{\lambda^{\prime}}$ as a functor from $C\left(\lambda^{\prime \prime}\right)$ to $C\left(\lambda^{\prime}\right)$. We will also show that $T_{\lambda^{\prime}, q}^{\lambda^{\prime \prime}}$ may be viewed as a functor from $C\left(\lambda^{\prime}\right)$ to $C\left(\lambda^{\prime \prime}\right)$.

We begin our study of the properties of translation by showing that modules with VS behave nicely under translation.

TheOREM 5.4. Let $M \in \mathrm{Ob} C\left(\lambda^{\prime}\right)$ have a $V S 0=M_{0} \subset M_{1} \subset \cdots$, let $N \in \mathrm{Ob} C\left(\lambda^{\prime}\right)$ have a $V S 0=N_{0} \subset N_{1} \subset \cdots$, and let $\phi \in \operatorname{Hom}(M, N)$.

(i) For each $n=0,1, \ldots$, the map

$$
\left(M_{n}^{\left(\lambda^{\prime}\right)} \otimes_{K} \Gamma\left(\lambda^{\prime \prime}-\lambda^{\prime}\right)\right)^{\left(\lambda^{\prime \prime}\right)} \rightarrow\left(M_{n+1}^{\left(\lambda^{\prime}\right)} \otimes_{K} \Gamma\left(\lambda^{\prime \prime}-\lambda^{\prime}\right)\right)^{\left(\lambda^{\prime \prime}\right)},
$$


which is induced by the inclusion $M_{n} \subset M_{n+1}$, is injective, and

$$
T_{\lambda^{\prime}}^{\lambda^{\prime \prime}} M=\underset{n}{\lim }\left(M_{n}^{\left(\lambda^{\prime}\right)} \otimes_{K} \Gamma\left(\lambda^{\prime \prime}-\lambda^{\prime}\right)\right)^{\left(\lambda^{\prime \prime}\right)} .
$$

Also, if $q>0$ then $T_{\lambda^{\prime}, q}^{\lambda^{\prime \prime}} M=0$.

(ii) For each $n$ let $k(n)$ be the smallest integer such that $\phi\left(M_{n}\right) \subseteq$ $N_{k(n)}$, and denote by $\phi_{n}: M_{n} \rightarrow N_{k(n)}$ the restriction of $\phi$. Then we have $T_{\lambda^{\prime}}^{\lambda^{\prime \prime}} \phi=\lim _{n}\left(\phi_{n}{ }^{\left(\lambda^{\prime}\right)} \otimes 1\right)^{\left(\lambda^{\prime \prime}\right)}$.

Proof. Since

$$
0 \rightarrow M_{n} \rightarrow M_{n+1} \rightarrow M_{n+1} / M_{n} \rightarrow 0
$$

is a short exact sequence in which all terms have a finite VS, by Proposition 4.20 we obtain the exactness of

$$
0 \rightarrow M_{n}^{\left(\lambda^{\prime}\right)} \rightarrow M_{n+1}^{\left(\lambda^{\prime}\right)} \rightarrow\left(M_{n+1} / M_{n}\right)^{\left(\lambda^{\prime}\right)} \rightarrow 0,
$$

and hence that of

$$
\begin{aligned}
0 & \rightarrow M_{n}^{\left(\lambda^{\prime}\right)} \otimes_{K} \Gamma\left(\lambda^{\prime \prime}-\lambda^{\prime}\right) \rightarrow M_{n+1}{ }^{\left(\lambda^{\prime}\right)} \otimes_{K} \Gamma\left(\lambda^{\prime \prime}-\lambda^{\prime}\right) \\
& \rightarrow\left(M_{n+1} / M_{n}\right)^{\left(\lambda^{\prime}\right)} \otimes_{K} \Gamma\left(\lambda^{\prime \prime}-\lambda^{\prime}\right) \rightarrow 0 .
\end{aligned}
$$

But, by Proposition 4.16, each of these modules has an RVS, so that by Theorem 4.19 we obtain the injectivity of

$$
\left(M_{n}^{\left(\lambda^{\prime}\right)} \otimes_{K} \Gamma\left(\lambda^{\prime \prime}-\lambda^{\prime}\right)\right)^{\left(\lambda^{\prime \prime}\right)} \rightarrow\left(M_{n+1}^{\left(\lambda^{\prime}\right)} \otimes_{K} \Gamma\left(\lambda^{\prime \prime}-\lambda^{\prime}\right)\right)^{\left(\lambda^{\prime \prime}\right)} .
$$

Now, for any $\mu \in \mathfrak{h}^{*}$, there is an integer $n(\mu)$ such that $M / M_{n(\mu)}$ has no weights $\geq \mu$. Thus, if we apply Lemma 4.8 to the finite VS (that is, finite RVS) of $M_{n(\mu)}$, we may reorder the factors of $M_{n(\mu)}$ if necessary to obtain a finite VS $0=M_{0}^{\prime} \subset M_{1}^{\prime} \subset \cdots \subset M_{n(\mu)}^{\prime}=M_{n(\mu)}$ such that for some $k(\mu) \in\{0,1, \ldots, n(\mu)\}$ we have that each factor of $0=M_{0}^{\prime} \subset M_{1}^{\prime} \subset \cdots \subset M_{k(\mu)}^{\prime}$ is of the form $M(\chi)$ for some $\chi \geq \mu$, and each factor of $M_{k(\mu)}^{\prime} \subset M_{k(\mu)+1}^{\prime} \subset \cdots \subset M_{n(\mu)}^{\prime}$ is of the form $M(\chi)$ for some $\chi \geq \mu$. Let

$$
\cdots \stackrel{d_{q+1}}{\rightarrow} P_{q} \stackrel{d_{q}}{\rightarrow} \ldots \stackrel{d_{1}}{\rightarrow} P_{0} \stackrel{d_{0}}{\rightarrow} M_{k(\mu)}^{\prime} \rightarrow 0
$$

be a projective resolution of $M_{k(\mu)}^{\prime}$ as in Proposition 3.11. Observe that this is in fact a local projective resolution of $M$ at $\mu$ when we replace $d_{0}$ by the composition of $d_{0}$ with the inclusion $M_{k(\mu)}^{\prime} \subseteq M$. By Proposition 3.11, all the images and kernels of the projective resolution have 
finite VS, so we may apply Theorem 4.19 twice and Proposition 4.16 once to obtain the exactness of

$$
\begin{aligned}
\cdots & \rightarrow\left(P_{q}^{\left(\lambda^{\prime}\right)} \otimes_{K} \Gamma\left(\lambda^{\prime \prime}-\lambda^{\prime}\right)\right)^{\left(\lambda^{\prime \prime}\right)} \rightarrow \cdots \rightarrow\left(P_{0}^{\left(\lambda^{\prime}\right)} \otimes_{K} \Gamma\left(\lambda^{\prime \prime}-\lambda^{\prime}\right)\right)^{\left(\lambda^{\prime \prime}\right)} \\
& \rightarrow\left(M_{k(\mu)}^{\prime\left(\lambda^{\prime}\right)} \otimes_{K} \Gamma\left(\lambda^{\prime \prime}-\lambda^{\prime}\right)\right)^{\left(\lambda^{\prime \prime}\right)} \rightarrow 0 .
\end{aligned}
$$

Now, letting $G$ be the functor used in Definition 5.3 to define the translation functors, we have that $\left(L_{q, \mu} G\right) M$ is the $q$ th homology of this complex with the last term deleted, so that by exactness we obtain $\left(L_{q, \mu} G\right) M=0$ for $q>0$ and

$$
\left(L_{0, \mu} G\right) M \cong\left(M_{k(\mu)}^{\prime}{ }^{\left(\lambda^{\prime}\right)} \otimes_{K} \Gamma\left(\lambda^{\prime \prime}-\lambda^{\prime}\right)\right)^{\left(\lambda^{\prime \prime}\right)} .
$$

Passing to the direct limit gives $T_{\lambda^{\prime}, q}^{\lambda^{\prime \prime}} M=0$ for $q>0$, and

$$
T_{\lambda^{\prime}}^{\lambda^{\prime \prime}} M=\underset{\mu}{\lim }\left(M_{k(\mu)}^{\prime}{ }^{\left(\lambda^{\prime}\right)} \otimes_{K} \Gamma\left(\lambda^{\prime \prime}-\lambda^{\prime}\right)\right)^{\left(\lambda^{\prime \prime}\right)} .
$$

Observe that if $\mu_{1} \geq \mu_{2}$, then $k\left(\mu_{2}\right) \geq k\left(\mu_{1}\right)$, so that choosing $\mu^{\prime} \in$ $\mathfrak{h}^{*}$ low enough we have $M_{k(\mu)}^{\prime} \subseteq M_{n(\mu)} \subseteq M_{k\left(\mu^{\prime}\right)}^{\prime}$. By the injectivity assertion which has already been proved, we have injections

$$
\begin{aligned}
\left(M_{k(\mu)}^{\prime}{ }^{\left(\lambda^{\prime}\right)}\right. & \left.\otimes_{K} \Gamma\left(\lambda^{\prime \prime}-\lambda^{\prime}\right)\right)^{\left(\lambda^{\prime \prime}\right)} \rightarrow\left(M_{n(\mu)}{ }^{\left(\lambda^{\prime}\right)} \otimes_{K} \Gamma\left(\lambda^{\prime \prime}-\lambda^{\prime}\right)\right)^{\left(\lambda^{\prime \prime}\right)} \\
& \rightarrow\left(M_{k\left(\mu^{\prime}\right)}^{\prime}\left(\lambda^{\prime}\right) \otimes_{K} \Gamma\left(\lambda^{\prime \prime}-\lambda^{\prime}\right)\right)^{\left(\lambda^{\prime \prime}\right)} .
\end{aligned}
$$

Since the injectivity also implies that

$$
\underset{n}{\lim }\left(M_{n}^{\left(\lambda^{\prime}\right)} \otimes_{K} \Gamma\left(\lambda^{\prime \prime}-\lambda^{\prime}\right)\right)^{\left(\lambda^{\prime \prime}\right)}
$$

is just the union of the images of the various $\left(M_{n}^{\left(\lambda^{\prime}\right)} \otimes_{K} \Gamma\left(\lambda^{\prime \prime}-\lambda^{\prime}\right)\right)^{\left(\lambda^{\prime \prime}\right)}$, we obtain

$$
\left.\underset{\mu}{\underset{\mu}{\lim }\left(M_{k(\mu)}^{\prime}\left(\lambda^{\prime}\right)\right.} \otimes_{K} \Gamma\left(\lambda^{\prime \prime}-\lambda^{\prime}\right)\right)^{\left(\lambda^{\prime \prime}\right)} \cong \underset{n}{\lim }\left(M_{n}^{\left(\lambda^{\prime}\right)} \otimes_{K} \Gamma\left(\lambda^{\prime \prime}-\lambda^{\prime}\right)\right)^{\left(\lambda^{\prime \prime}\right)},
$$

and hence

$$
T_{\lambda^{\prime}}^{\lambda^{\prime \prime}} M \cong \underset{n}{\lim }\left(M_{n}^{\left(\lambda^{\prime}\right)} \otimes_{K} \Gamma\left(\lambda^{\prime \prime}-\lambda^{\prime}\right)\right)^{\left(\lambda^{\prime \prime}\right)},
$$

proving (i).

For statement (ii) on maps, we again begin by considering $L_{q, \mu} G$ for some $\mu \in \mathfrak{h}^{*}$. Choosing $m, n$ so that $M / M_{m}$ and $N / N_{n}$ have no weights $\geq \mu$, we may repeat the above argument to obtain projective resolutions

$$
\cdots \rightarrow P_{q} \rightarrow \cdots \rightarrow P_{0} \rightarrow M_{k(\mu)}^{\prime} \rightarrow 0
$$


and

$$
\cdots \rightarrow Q_{q} \rightarrow \cdots \rightarrow Q_{0} \rightarrow N_{k^{\prime}(\mu)}^{\prime} \rightarrow 0
$$

which can be considered as local projective resolutions of $M$ and $N$, respectively, at $\mu$. Clearly $\phi\left(M_{k(\mu)}^{\prime}\right) \subseteq N_{k^{\prime}(\mu)}$ by considering the highest weight vectors of the Verma factors of $M_{k(\mu)}^{\prime}$. Thus, by the projectivity, we may obtain a ladder with exact rows

$$
\begin{gathered}
\cdots \rightarrow P_{q} \rightarrow \cdots \rightarrow P_{0} \rightarrow M_{k(\mu)}^{\prime} \rightarrow 0 \\
\downarrow \\
\cdots \rightarrow Q_{q} \rightarrow \cdots \rightarrow Q_{0} \rightarrow N_{k^{\prime}(\mu)}^{\prime} \rightarrow 0,
\end{gathered}
$$

so that applying the functor $\left(-\left(\lambda^{\prime}\right) \otimes_{K} \Gamma\left(\lambda^{\prime \prime}-\lambda^{\prime}\right)\right)^{\left(\lambda^{\prime \prime}\right)}$ and passing to homology we see that $\left(L_{0, \mu} G\right) \phi$ is just the map induced by $\phi_{k(\mu)}$ on the tensor product, that is $\left(\phi_{k(\mu)}{ }^{\left(\lambda^{\prime}\right)} \otimes 1\right)^{\left(\lambda^{\prime \prime}\right)}$. Passage to the direct limit gives the result.

For the translation functors $T_{\lambda^{\prime \prime}, q}^{\lambda^{\prime}}$, we can prove a similar theorem, using the same method. In this case, however, the statement is easier, since when $M \in \mathrm{Ob} C\left(\lambda^{\prime \prime}\right)$ has a VS $0=M_{0} \subset M_{1} \subset \cdots$, so does $M^{\left(\lambda^{\prime \prime}\right)} \otimes_{K} L\left(\lambda^{\prime}-\lambda^{\prime \prime}\right)$, and in fact this latter module is an object in $C\left(\lambda^{\prime}\right)$, hence so is $\left(M^{\left(\lambda^{\prime \prime}\right)} \otimes_{K} L\left(\lambda^{\prime}-\lambda^{\prime \prime}\right)\right)^{\left(\lambda^{\prime}\right)}$, and we easily have

$$
\left(M^{\left(\lambda^{\prime \prime}\right)} \otimes_{K} L\left(\lambda^{\prime}-\lambda^{\prime \prime}\right)\right)^{\left(\lambda^{\prime}\right)} \cong \underset{n}{\lim }\left(M_{n}{ }^{\left(\lambda^{\prime \prime}\right)} \otimes_{K} L\left(\lambda^{\prime}-\lambda^{\prime \prime}\right)\right)^{\left(\lambda^{\prime}\right)},
$$

so that we need not write the direct limit in the statement of the theorem. We omit the proof, however, since it is essentially the same as that of Theorem 5.4.

Theorem 5.5. Let $M \in \mathrm{Ob} C\left(\lambda^{\prime \prime}\right)$ have a VS. Then

$$
T_{\lambda^{\prime \prime}}^{\lambda^{\prime}} M \cong\left(M^{\left(\lambda^{\prime \prime}\right)} \otimes_{K} L\left(\lambda^{\prime}-\lambda^{\prime \prime}\right)\right)^{\left(\lambda^{\prime}\right)}
$$

and $T_{\lambda^{\prime \prime}, q}^{\lambda^{\prime}} M=0$ for all $q>0$. Furthermore, if $N \in \mathrm{Ob} C\left(\lambda^{\prime \prime}\right)$ also has $a V S$ and $\phi \in \operatorname{Hom}(M, N)$, then $T_{\lambda^{\prime \prime}}^{\lambda^{\prime}} \phi=\left(\phi^{\left(\lambda^{\prime \prime}\right)} \otimes 1\right)^{\left(\lambda^{\prime}\right)}$.

Corollary 5.6. Let $M \in \mathrm{Ob} C\left(\lambda^{\prime}\right)$ (respectively, $C\left(\lambda^{\prime \prime}\right)$ ), and suppose we have an exact resolution

$$
\cdots \rightarrow M_{q} \rightarrow \cdots \rightarrow M_{0} \rightarrow M \rightarrow 0
$$

in $C\left(\lambda^{\prime}\right)$ (respectively, $C\left(\lambda^{\prime \prime}\right)$ ) such that each $M_{q}$ has a VS. Then $T_{\lambda^{\prime}, q}^{\lambda^{\prime \prime}} M$ $\cong H_{q}\left(T_{\lambda^{\prime}}^{\lambda^{\prime \prime}} M_{*}\right)$ (respectively, $T_{\lambda^{\prime \prime}, q}^{\lambda^{\prime}} M \cong H_{q}\left(T_{\lambda^{\prime \prime}}^{\lambda^{\prime}} M_{*}\right)$ ).

Proof. This follows from Theorems 5.4 and 5.5, by the Acyclic Model Theorem. 
Observe that for any $\mu \in \mathfrak{h}^{*}$ and any $M \in \mathrm{Ob} C(\mu), M$ has an exact resolution

$$
\cdots \rightarrow M_{q} \rightarrow \cdots \rightarrow M_{0} \rightarrow M \rightarrow 0
$$

in $C(\mu)$ in which each $M_{q}$ has a VS. To see this, simply tensor the resolution of the trivial module

$\cdots \rightarrow U(\mathfrak{g}) \otimes_{U(\mathfrak{b})}\left(\bigwedge^{q}(\mathfrak{g} / \mathfrak{b})\right) \rightarrow \cdots \rightarrow U(\mathfrak{g}) \otimes_{U(\mathfrak{b})}\left(\bigwedge^{0}(\mathfrak{g} / \mathfrak{b})\right) \rightarrow K \rightarrow 0$

with $M$ over $K$. By [5, Proposition 1.7] we have that

$$
\left(U(\mathfrak{g}) \otimes_{U(\mathfrak{b})}\left(\bigwedge^{q}(\mathfrak{g} / \mathfrak{b})\right)\right) \otimes_{K} \cong U(\mathfrak{g}) \otimes_{U(\mathfrak{b})}\left(\bigwedge^{q}(\mathfrak{g} / \mathfrak{b}) \otimes_{K} M\right),
$$

and thus an easy application of Proposition 4.3 shows that this module has a VS. Thus, resolutions satisfying the hypotheses of Corollary 5.6 exist.

Using the existence of such a resolution for any $M \in \mathrm{Ob} C\left(\lambda^{\prime \prime}\right)$ and Corollary 5.6 together with Theorem 5.5 gives the following result.

Corollary 5.7. For any $M \in \mathrm{Ob} C\left(\lambda^{\prime \prime}\right)$ and any $q>0, T_{\lambda^{\prime \prime}, q}^{\lambda^{\prime}} M$ is an object of $C\left(\lambda^{\prime}\right)$.

In view of this corollary, we will henceforth regard the functors $T_{\lambda^{\prime \prime}, q}^{\lambda^{\prime}}$ as functors from $C\left(\lambda^{\prime \prime}\right)$ to $C\left(\lambda^{\prime}\right)$. Note that we cannot as yet make the similar claim for $T_{\lambda^{\prime}, q}^{\lambda^{\prime \prime}}$, since in the complex used to compute this functor in Corollary 5.6 we are dealing with a direct limit, which is not necessarily an object of $C\left(\lambda^{\prime \prime}\right)$.

We now turn to some explicit computations of the translations of Verma modules.

Proposition 5.8. Let $W_{\lambda^{\prime}}$ (respectively, $\left.W_{\lambda^{\prime \prime}}\right)$ denote the stabilizer of $\lambda^{\prime}$ (respectively, $\lambda^{\prime \prime}$ ) in $W$ under the dot action, and let $X$ be a set of left coset representatives for $W_{\lambda^{\prime}}$ in $W_{\lambda^{\prime \prime}}$. If $w \in W$, then $T_{\lambda^{\prime \prime}}^{\lambda^{\prime}} M\left(w \cdot \lambda^{\prime \prime}\right)$ has a VS with factors of the form $M\left((w x) \cdot \lambda^{\prime}\right)$ with $x \in X$, and each of these factors occurs with multiplicity one.

Proof. Since

$$
M\left(w \cdot \lambda^{\prime \prime}\right)^{\left(\lambda^{\prime \prime}\right)} \cong M\left(w \cdot \lambda^{\prime \prime}\right) \text { and } M\left(w \cdot \lambda^{\prime \prime}\right) \otimes_{K} L\left(\lambda^{\prime}-\lambda^{\prime \prime}\right)
$$

has a VS, by Proposition 4.17, in which the multiplicity of any $M(\zeta)$ is equal to $\operatorname{dim} L\left(\lambda^{\prime}-\lambda^{\prime \prime}\right)_{\zeta-w \cdot \lambda^{\prime \prime}}$, we have that for any $M(\zeta)$ occurring 
in the VS and any $y \in W$, that $y\left(\zeta-w \cdot \lambda^{\prime \prime}\right) \leq \lambda^{\prime}-\lambda^{\prime \prime}$. In particular, if $M\left(w^{\prime} \cdot \lambda^{\prime}\right)$ occurs then $w^{\prime-1}\left(w^{\prime} \cdot \lambda^{\prime}-w \cdot \lambda^{\prime \prime}\right)=\lambda^{\prime}-\left(w^{\prime-1} w\right) \cdot \lambda^{\prime \prime} \leq \lambda^{\prime}-\lambda^{\prime \prime}$, so that we must have $\left(w^{\prime-1} w\right) \cdot \lambda^{\prime \prime}=\lambda^{\prime \prime}$, since $\lambda^{\prime \prime}+\rho \in P^{+}$. By Theorem 5.5, $T_{\lambda^{\prime \prime}}^{\lambda^{\prime}} M\left(w \cdot \lambda^{\prime \prime}\right)$ has a VS, and all of its factors are of the form $M\left(w^{\prime} \cdot \lambda^{\prime \prime}\right)$ for some $w^{\prime} \in W$. But we have just observed that any such factor satisfies $w^{\prime-1} w \in W_{\lambda^{\prime \prime}}$, so that $w^{\prime}=w x$ for some $x \in W_{\lambda^{\prime \prime}}$. That is, for any factor $M\left(w^{\prime} \cdot \lambda^{\prime}\right)$ which occurs, we have $w^{\prime} \cdot \lambda^{\prime}=(w x) \cdot \lambda^{\prime}$ for some $x \in W_{\lambda^{\prime \prime}}$. Note that to obtain all weights of the form $(w x) \cdot \lambda^{\prime}$ with $x \in W_{\lambda^{\prime \prime}}$, we need only choose $x \in X$, a set of left coset representatives for $W_{\lambda^{\prime}}$ in $W_{\lambda^{\prime \prime}}$, since if $y \in W_{\lambda^{\prime}}$ then $(w x y) \cdot \lambda^{\prime}=(w x) \cdot \lambda^{\prime}$. It remains to prove the multiplicity assertion. We have that the multiplicity of $M\left((w x) \cdot \lambda^{\prime}\right)$ is equal to

$$
\begin{aligned}
\operatorname{dim} & L\left(\lambda^{\prime}-\lambda^{\prime \prime}\right)_{(w x) \cdot \lambda^{\prime}-w \cdot \lambda^{\prime \prime}} \\
& =\operatorname{dim} L\left(\lambda^{\prime}-\lambda^{\prime \prime}\right)_{(w x)^{-1}\left((w x) \cdot \lambda^{\prime}-w \cdot \lambda^{\prime \prime}\right)} \\
& =\operatorname{dim} L\left(\lambda^{\prime}-\lambda^{\prime \prime}\right)_{\lambda^{\prime}-x^{-1} \cdot \lambda^{\prime \prime}}=\operatorname{dim} L\left(\lambda^{\prime}-\lambda^{\prime \prime}\right)_{\lambda^{\prime}-\lambda^{\prime \prime}}=1,
\end{aligned}
$$

since $x^{-1} \in W_{\lambda^{\prime \prime}}$

Proposition 5.9. Let $w \in W$. Then $T_{\lambda^{\prime}}^{\lambda^{\prime \prime}} M\left(w \cdot \lambda^{\prime}\right) \cong M\left(w \cdot \lambda^{\prime \prime}\right)$.

Proof. Note that $M\left(w \cdot \lambda^{\prime}\right)^{\left(\lambda^{\prime}\right)} \cong M\left(w \cdot \lambda^{\prime}\right)$. By Theorem 5.4, we therefore need only consider the Verma factors of $M\left(w \cdot \lambda^{\prime}\right) \otimes_{K}$ $\Gamma\left(\lambda^{\prime \prime}-\lambda^{\prime}\right)$ which are of the form $M\left(w^{\prime} \cdot \lambda^{\prime \prime}\right)$ for some $w^{\prime} \in W$. Now, by Proposition 4.16 $M\left(w \cdot \lambda^{\prime}\right) \otimes_{K} \Gamma\left(\lambda^{\prime \prime}-\lambda^{\prime}\right)$ has an RVS in which the multiplicity of any $M(\zeta)$ is equal to $\operatorname{dim} \Gamma\left(\lambda^{\prime \prime}-\lambda^{\prime}\right)_{\zeta-w \cdot \lambda^{\prime}}$, so that for any factor $M(\zeta)$ which occurs in the RVS we have for all $x \in W$ that $x\left(\zeta-w \cdot \lambda^{\prime}\right) \geq \lambda^{\prime \prime} \geq \lambda^{\prime}$. In particular, if $M\left(w^{\prime} \cdot \lambda^{\prime \prime}\right)$ occurs, then $w^{-1}\left(w^{\prime} \cdot \lambda^{\prime \prime}-w \cdot \lambda^{\prime}\right)=\left(w^{-1} w^{\prime}\right) \cdot \lambda^{\prime \prime}-\lambda^{\prime} \geq \lambda^{\prime \prime}-\lambda^{\prime}$, so that $\left(w^{-1} w^{\prime}\right) \cdot \lambda^{\prime \prime} \geq \lambda^{\prime \prime}$, and, since $\lambda^{\prime \prime}+\rho \in P^{+},\left(w^{-1} w^{\prime}\right) \cdot \lambda^{\prime \prime}=\lambda^{\prime \prime}$. This shows that for any factor $M\left(w^{\prime} \cdot \lambda^{\prime \prime}\right)$ which occurs, we must have that $w^{\prime} \cdot \lambda^{\prime \prime}=w \cdot \lambda^{\prime \prime}$, so that $M\left(w \cdot \lambda^{\prime \prime}\right)$ is the only Verma factor which occurs in the finite RVS for $T_{\lambda^{\prime}}^{\lambda^{\prime \prime}} M\left(w \cdot \lambda^{\prime}\right)$. Its multiplicity is

$$
\begin{aligned}
\operatorname{dim} \Gamma\left(\lambda^{\prime \prime}-\lambda^{\prime}\right)_{w \cdot \lambda^{\prime \prime}-w \cdot \lambda^{\prime}} & =\operatorname{dim} \Gamma\left(\lambda^{\prime \prime}-\lambda^{\prime}\right)_{w^{-1}\left(w \cdot \lambda^{\prime \prime}-w \cdot \lambda^{\prime}\right)} \\
& =\operatorname{dim} \Gamma\left(\lambda^{\prime \prime}-\lambda^{\prime}\right)_{\lambda^{\prime \prime}-\lambda^{\prime}}=1,
\end{aligned}
$$

and we have that $T_{\lambda^{\prime}}^{\lambda^{\prime \prime}} M\left(w \cdot \lambda^{\prime}\right) \cong M\left(w \cdot \lambda^{\prime \prime}\right)$.

REMARK. The assumption that $W_{\lambda^{\prime}}$ have finite index in $W_{\lambda^{\prime \prime}}$ was not needed for the preceding two propositions. Also, similar results were obtained in [4] for the case of a symmetrizable GCM. 
Corollary 5.10. If $M \in \mathrm{Ob} C\left(\lambda^{\prime}\right)$ has a VS, then $T_{\lambda^{\prime}}^{\lambda^{\prime \prime}} M$ is an object of $C\left(\lambda^{\prime \prime}\right)$ and it has a VS whose factors are all of the form $M\left(w \cdot \lambda^{\prime \prime}\right)$ for some $w \in W$.

Proof. Let $0=M_{0} \subset M_{1} \subset \cdots$ be a VS for $M$, so that by Theorem 5.4

$$
T_{\lambda^{\prime}}^{\lambda^{\prime \prime}} M=\underset{n}{\lim }\left(M_{n}^{\left(\lambda^{\prime}\right)} \otimes_{K} \Gamma\left(\lambda^{\prime \prime}-\lambda^{\prime}\right)\right)^{\left(\lambda^{\prime \prime}\right)},
$$

with the maps being injective. Identifying each $\left(M_{n}{ }^{\left(\lambda^{\prime}\right)} \otimes_{K} \Gamma\left(\lambda^{\prime \prime}-\lambda^{\prime}\right)\right)^{\left(\lambda^{\prime \prime}\right)}$ with its image $T_{n}$ in the direct limit $T_{\lambda^{\prime}}^{\lambda^{\prime \prime}} M$, we have that $0=T_{0} \subset$ $T_{1} \subset \cdots$ and $T_{\lambda^{\prime}}^{\lambda^{\prime \prime}} M=\bigcup_{n \geq 0} T_{n}$. By Theorem 4.19 and Propositions 4.20 and 5.9, $T_{n+1} / T_{n} \cong M\left(w \cdot \lambda^{\prime \prime}\right)$ if $M_{n+1} / M_{n} \cong M\left(w \cdot \lambda^{\prime}\right)$, and $T_{n+1} / T_{n}=0$ if $M_{n+1} / M_{n} \cong M(\mu)$ with $\mu \notin W \cdot \lambda^{\prime}$. Note also that each $M\left(w \cdot \lambda^{\prime}\right)$ can occur only finitely many times in the VS for $M$, so that $M\left(w \cdot \lambda^{\prime \prime}\right)$ occurs only finitely many times as a factor in the filtration $0=T_{0} \subset T_{1} \subset \cdots$ of $T_{\lambda^{\prime}}^{\lambda^{\prime \prime}} M$. Eliminating repeated terms in the filtration gives the result.

REMARK. It is in the above corollary where the assumption that $W_{\lambda^{\prime}}$ have finite index in $W_{\lambda^{\prime \prime}}$ is necessary. For example, with $W$ infinite, take $\lambda^{\prime}=0$ and $\lambda^{\prime \prime}=-\rho$, so that $W_{\lambda^{\prime}}=\{1\}$ and $W_{\lambda^{\prime \prime}}=W$. If $M=$ $\bigoplus_{w \in W} M(w \cdot 0) \in \mathrm{Ob} C(0)$, then $T_{0}^{-\rho} M \cong \bigoplus_{w \in W} M(w \cdot(-\rho))$ is not an object of $C(-\rho)$, since $w \cdot(-\rho)=-\rho$ for all $w \in W$, and hence all the weight spaces of $T_{0}^{-\rho} M$ are infinite dimensional. Thus, Corollary 5.10 is not true without the finite index assumption, and we would also not be able to obtain the next result without this assumption.

Corollary 5.11. For any $M \in \mathrm{Ob} C\left(\lambda^{\prime}\right)$ and any $q \geq 0, T_{\lambda^{\prime}, q}^{\lambda^{\prime \prime}} M$ is an object of $C\left(\lambda^{\prime \prime}\right)$, and furthermore any composition factor $L(\mu)$ of $T_{\lambda^{\prime}, q}^{\lambda^{\prime \prime}} M$ satisfies $w \cdot \mu \leq \lambda^{\prime \prime}$ for all $w \in W$.

Proof. Let

$$
\cdots \rightarrow M_{q} \rightarrow \cdots \rightarrow M_{0} \rightarrow M \rightarrow 0
$$

be an exact resolution of $M$ in $C\left(\lambda^{\prime}\right)$ such that each $M_{q}$ has a VS. By Corollary 5.10, each $T_{\lambda^{\prime}}^{\lambda^{\prime \prime}} M_{q}$ is an object in $C\left(\lambda^{\prime \prime}\right)$ with a VS whose factors are of the form $M\left(w \cdot \lambda^{\prime \prime}\right)$ for some $w \in W$. The first statement now follows from Corollary 5.6, and since any composition factor of $T_{\lambda^{\prime}, q}^{\lambda^{\prime \prime}} M$ is also a composition factor of $T_{\lambda^{\prime}}^{\lambda^{\prime \prime}} M_{q}$, the second statement follows from Propositions 4.5 and 5.1.

In view of this corollary, we will henceforth view the translation functors $T_{\lambda^{\prime}, q}^{\lambda^{\prime \prime}}$ as functors from $C\left(\lambda^{\prime}\right)$ to $C\left(\lambda^{\prime \prime}\right)$. 
6. A spectral sequence for $T_{\lambda^{\prime \prime}, q}^{\lambda^{\prime}}$. We construct a spectral sequence converging to $T_{\lambda^{\prime \prime}, q}^{\lambda^{\prime}} M$, for any $M \in \mathrm{Ob} C\left(\lambda^{\prime \prime}\right)$. Using this spectral sequence, we show for the case $q=0$ that the translation functor $T_{\lambda^{\prime \prime}}^{\lambda^{\prime}}$ may be obtained as the composition of three functors. This fact will be important in the next section, where it will be used to prove an adjoint-like property of the translation functors.

Definition 6.1. In any category of the form $C(\mu)$ with $\mu \in \mathfrak{h}^{*}$, and for any $\nu \in \mathfrak{h}^{*}$ with $\nu+\rho \in P^{+}$, we define $W_{q}^{\nu}(q=0,1, \ldots)$ to be the $q$ th left derived functor of the functor $M \mapsto M^{(\nu)}$ from the full subcategory of $C(\mu)$ whose objects are finite direct sums of various $I^{\mu}(\chi)$ to the category of weight modules.

REMARK. The functors $W_{q}^{\nu}$ were introduced in [11], where it was shown that they do not depend on the choice of $\mu$, provided $M \in$ $\mathrm{Ob} C(\mu)$, so that we simply write $W_{q}^{\nu} M$ without possibility of confusion.

We now summarize several properties of the functors $W_{q}^{\nu}$ which were proved in [11].

Proposition 6.2 [11, Theorem 6.2 and Lemma 6.6]. Let $\mu \in \mathfrak{h}^{*}$, $\nu+\rho \in P^{+}$, and $M \in \mathrm{Ob} C(\mu)$. If

$$
\cdots \rightarrow M_{q} \rightarrow \cdots \rightarrow M_{0} \rightarrow M \rightarrow 0
$$

is an exact resolution of $M$ in $C(\mu)$ where each $M_{q}$ has a VS, then $W_{q}^{\nu} M \cong H_{q}\left(M_{*}^{(\nu)}\right)$. In particular, if $M$ has a $V S$, then $W_{q}^{\nu} M=0$ for $q>0$ and $W_{0}^{\nu} M \cong M^{(\nu)}$. Furthermore, $W_{0}^{\nu} L(\chi)=0$ if $\chi \notin W \cdot \nu$, and for each $w \in W$ there is an epimorphism $\pi: W_{0}^{\nu} L(w \cdot \nu) \rightarrow L(w \cdot \nu)$ such that every composition factor $L(\zeta)$ of $\operatorname{Ker} \pi$ satisfies $x \cdot \zeta<\nu$ for all $x \in W$.

We now come to the main result of this section, which is essentially a Grothendieck spectral sequence. In the proof, however, we use resolutions by modules with VS, rather than proper projective resolutions of complexes. With this change, our proof is an imitation of the proof of [14, Theorem 11.38]. We use the notation and terminology of [14] for filtrations on bicomplexes and the associated total complexes and 
spectral sequences. In particular, for any bicomplex

$$
\begin{aligned}
& \begin{array}{rr}
0 & 0 \\
d_{10}^{\prime \prime} \uparrow & d_{00}^{\prime \prime} \uparrow
\end{array} \\
& \cdots \rightarrow \underset{d_{11}^{\prime \prime} \uparrow}{M_{10}} \stackrel{d_{10}^{\prime}}{\rightarrow} \underset{d_{01}^{\prime \prime} \uparrow}{M_{00}} \stackrel{d_{00}^{\prime}}{\rightarrow} 0
\end{aligned}
$$

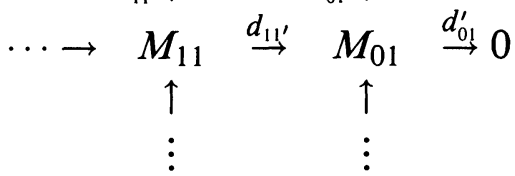

(that is, each row and each column a complex and $d_{p-1, q}^{\prime \prime} d_{p q}^{\prime}+d_{p, q-1}^{\prime} d_{p q}^{\prime \prime}$ $=0$ for all $p$ and $q)$ we use the notations $H_{p q}^{\prime}\left(M_{* *}\right)$ for Ker $d_{p q}^{\prime} / \operatorname{Im} d_{p+1, q}^{\prime}$ and $H_{p q}^{\prime \prime}\left(M_{* *}\right)$ for $\operatorname{Ker} d_{p q}^{\prime \prime} / \operatorname{Im} d_{p, q+1}^{\prime \prime}$. Also the total complex Tot $M$ is the complex with $n$th term $\bigoplus_{p+q=n} M_{p q}$ and differential $d^{\prime}+d^{\prime \prime}$.

THEOREM 6.3. For any $M \in \mathrm{Ob} C\left(\lambda^{\prime \prime}\right)$, there is a third quadrant spectral sequence with $E_{p q}^{2} \cong W_{p}^{\lambda^{\prime}}\left[W_{q}^{\lambda^{\prime \prime}} M \otimes_{K} L\left(\lambda^{\prime}-\lambda^{\prime \prime}\right)\right] \underset{p}{\Rightarrow} T_{\lambda^{\prime \prime}, p+q}^{\lambda^{\prime}} M$.

Proof. Recall the standard resolution of the trivial module

$$
\cdots \rightarrow U(\mathfrak{g}) \otimes_{U(\mathfrak{b})} \bigwedge^{n}(\mathfrak{g} / \mathfrak{b}) \rightarrow \cdots \rightarrow U(\mathfrak{g}) \otimes_{U(\mathfrak{b})} \bigwedge^{0}(\mathfrak{g} / \mathfrak{b}) \rightarrow K \rightarrow 0 .
$$

By tensoring this resolution over $K$ with $M$ and applying [5, Proposition 1.7], we obtain a resolution

$$
\cdots \rightarrow M_{1} \rightarrow M_{0} \rightarrow M \rightarrow 0
$$

of $M$ in $C\left(\lambda^{\prime \prime}\right)$ by modules with VS, and hence we obtain a complex

$$
\cdots \rightarrow M_{1}^{\left(\lambda^{\prime \prime}\right)} \rightarrow M_{0}^{\left(\lambda^{\prime \prime}\right)} \rightarrow 0
$$

such that $H_{n}\left(M_{*}{ }^{\left(\lambda^{\prime \prime}\right)}\right) \cong W_{n}^{\lambda^{\prime \prime}} M$ for all $n \geq 0$, by Proposition 6.2. By again tensoring with the standard resolution and applying [5, Proposition 1.7], we obtain a bigraded module with commuting squares

$$
\begin{aligned}
& \begin{array}{cc}
0 & 0 \\
\uparrow & \uparrow \\
\ldots \rightarrow & M_{10} \rightarrow \\
M_{00} \rightarrow 0
\end{array} \\
& \stackrel{\uparrow}{\uparrow} \stackrel{\uparrow}{\uparrow} M_{11} \rightarrow M_{01} \rightarrow 0
\end{aligned}
$$


with $M_{p q}=U(\mathfrak{g}) \otimes_{U(\mathfrak{b})}\left[\bigwedge^{q}(\mathfrak{g} / \mathfrak{b}) \otimes_{K} M_{p}^{\left(\lambda^{\prime \prime}\right)}\right]$, so that each $M_{p q}$ has a VS by Proposition 4.3, and using a sign change for the maps on alternate rows, we obtain a bicomplex.

We note here that by the exactness of the functor

$$
\left[U(\mathfrak{g}) \otimes_{U(\mathfrak{b})} \bigwedge^{q}(\mathfrak{g} / \mathfrak{b})\right] \otimes_{K}-
$$

we obtain

$$
\begin{aligned}
& \operatorname{Ker}\left(M_{p q} \rightarrow M_{p-1, q}\right) \\
& \quad \cong U(\mathfrak{g}) \otimes_{U(\mathfrak{b})}\left[\bigwedge^{q}(\mathfrak{g} / \mathfrak{b}) \otimes_{K} \operatorname{Ker}\left(M_{p}^{\left.\lambda^{\prime \prime}\right)} \rightarrow M_{p-1}{ }^{\left(\lambda^{\prime \prime}\right)}\right)\right], \\
& \operatorname{Im}\left(M_{p+1, q} \rightarrow M_{p q}\right) \\
& \quad \cong U(\mathfrak{g}) \otimes_{U(\mathfrak{b})}\left[\bigwedge^{q}(\mathfrak{g} / \mathfrak{b}) \otimes_{K} \operatorname{Im}\left(M_{p+1}{ }^{\left(\lambda^{\prime \prime}\right)} \rightarrow M_{p}{ }^{\left(\lambda^{\prime \prime}\right)}\right)\right],
\end{aligned}
$$

and

$$
H_{p q}^{\prime}\left(M_{* *}\right)=U(\mathfrak{g}) \otimes_{U(\mathfrak{b})}\left[\bigwedge^{q}(\mathfrak{g} / \mathfrak{b}) \otimes_{K} H_{p}\left(M_{*}{ }^{\left(\lambda^{\prime \prime}\right)}\right)\right] .
$$

Thus each of these modules has a VS by Proposition 4.3.

Now, let $N_{p q}=\left(M_{p q} \otimes_{K} L\left(\lambda^{\prime}-\lambda^{\prime \prime}\right)\right)^{\left(\lambda^{\prime}\right)}$ for each $p, q$, and consider the third quadrant bicomplex

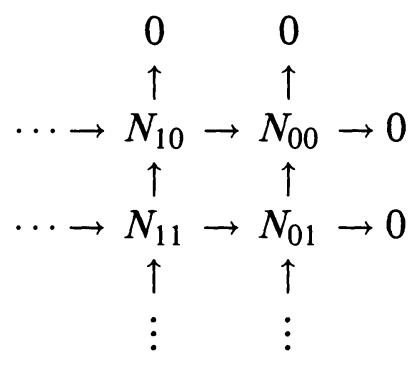

There are two natural filtrations of the total complex Tot $N$ associated to this bicomplex, the first obtained by restricting to certain columns of the bicomplex, and the second by restricting to certain rows. The associated spectral sequences have $E_{p q}^{2}$ terms given by $H_{p}^{\prime}\left(H_{p q}^{\prime \prime}\left(N_{* *}\right)\right)$ and $H_{p}^{\prime \prime}\left(H_{p q}^{\prime}\left(N_{* *}\right)\right)$, respectively, as shown in [14, Theorems 11.18 and 11.19]. This gives us two ways to compute $H_{n}(\operatorname{Tot} N)$, and it is the comparison of these two computations which will prove the theorem.

For the spectral sequence arising from the first filtration, since $H_{p q}^{\prime \prime}\left(N_{* *}\right)$ is the $q$ th homology of the complex

$$
\cdots \rightarrow\left(M_{p i} \otimes_{K} L\left(\lambda^{\prime}-\lambda^{\prime \prime}\right)\right)^{\left(\lambda^{\prime}\right)} \rightarrow \cdots \rightarrow\left(M_{p 0} \otimes_{K} L\left(\lambda^{\prime}-\lambda^{\prime \prime}\right)\right)^{\left(\lambda^{\prime}\right)} \rightarrow 0,
$$


and since

$$
\cdots \rightarrow M_{p i} \otimes_{K} L\left(\lambda^{\prime}-\lambda^{\prime \prime}\right) \rightarrow \cdots \rightarrow M_{p 0} \otimes_{K} L\left(\lambda^{\prime}-\lambda^{\prime \prime}\right) \rightarrow 0
$$

is a resolution of $M_{p}{ }^{\left(\lambda^{\prime \prime}\right)} \otimes_{K} L\left(\lambda^{\prime}-\lambda^{\prime \prime}\right)$ by modules each of which has a VS by Proposition 4.17, we have that by Proposition $6.2 H_{p q}^{\prime \prime}\left(N_{* *}\right) \cong$ $W_{q}^{\lambda^{\prime}}\left[M_{p}\left(\lambda^{\prime \prime}\right) \otimes_{K} L\left(\lambda^{\prime}-\lambda^{\prime \prime}\right)\right]$. But $M_{p}^{\left(\lambda^{\prime}\right)} \otimes_{K} L\left(\lambda^{\prime}-\lambda^{\prime \prime}\right)$ is itself a module with VS, so Proposition 6.2 gives

$$
H_{p q}^{\prime \prime}\left(N_{* *}\right)= \begin{cases}{\left[M_{p}^{\left(\lambda^{\prime \prime}\right)} \otimes_{K} L\left(\lambda^{\prime}-\lambda^{\prime \prime}\right)\right]^{\left(\lambda^{\prime}\right)},} & \text { if } q=0, \\ 0, & \text { if } q>0 .\end{cases}
$$

Thus, this spectral sequence collapses, and $E_{p 0}^{2}=H_{p}^{\prime}\left(H_{p 0}^{\prime \prime}\left(N_{* *}\right)\right) \cong$ $H_{p}\left(\left[M_{*}^{\left(\lambda^{\prime \prime}\right)} \otimes_{K} L\left(\lambda^{\prime}-\lambda^{\prime \prime}\right)\right]^{\left(\lambda^{\prime}\right)}\right) \cong T_{\lambda^{\prime \prime}, p}^{\lambda^{\prime}} M$ by Corollary 5.6. This shows that $H_{n}($ Tot $N) \cong T_{\lambda^{\prime \prime}, n}^{\lambda^{\prime}} M$.

On the other hand, for the spectral sequence arising from the second filtration, consider $H_{q p}^{\prime}\left(N_{* *}\right)=H_{q p}^{\prime}\left(\left[M_{* *} \otimes_{K} L\left(\lambda^{\prime}-\lambda^{\prime \prime}\right)\right]^{\left(\lambda^{\prime}\right)}\right)$. We have already seen that the rows

$$
\cdots \rightarrow M_{q+1, p} \rightarrow M_{q p} \rightarrow M_{q-1} \rightarrow \cdots \rightarrow M_{0 p} \rightarrow 0
$$

of the bicomplex $\left(M_{* *}\right)$ are complexes in which each term has a VS, and in which moreover each kernel, image, and homology has a VS. Thus, by Proposition 4.17, using the exactness of $-\otimes_{K} L\left(\lambda^{\prime}-\lambda^{\prime \prime}\right)$, the same is true of the complex

$$
\cdots \rightarrow M_{q p} \otimes_{K} L\left(\lambda^{\prime}-\lambda^{\prime \prime}\right) \rightarrow \cdots \rightarrow M_{0 p} \otimes_{K} L\left(\lambda^{\prime}-\lambda^{\prime \prime}\right) \rightarrow 0 .
$$

Using this fact together with the exactness of the functor $V \mapsto V^{\left(\lambda^{\prime}\right)}$ on short exact sequences of modules with VS stated in Proposition 4.20, we obtain

$$
H_{q p}^{\prime}\left(N_{* *}\right)=H_{q p}^{\prime}\left(\left[M_{* *} \otimes_{K} L\left(\lambda^{\prime}-\lambda^{\prime \prime}\right)\right]^{\left(\lambda^{\prime}\right)}\right) \cong\left[H_{q p}^{\prime}\left(M_{* *}\right) \otimes_{K} L\left(\lambda^{\prime}-\lambda^{\prime \prime}\right)\right]^{\left(\lambda^{\prime}\right)} \text {. }
$$

Since the sequence

$$
\cdots \rightarrow H_{q p}^{\prime}\left(M_{* *}\right) \rightarrow \cdots \rightarrow H_{0 p}^{\prime}\left(M_{* *}\right) \rightarrow H_{q}\left(M_{*}{ }^{\left(\lambda^{\prime \prime}\right)}\right) \rightarrow 0
$$

is an exact resolution by modules with VS as noted in the remarks following the definition of $M_{p q}$, we see that

$$
\begin{aligned}
\cdots & \rightarrow H_{q p}^{\prime}\left(M_{* *}\right) \otimes_{K} L\left(\lambda^{\prime}-\lambda^{\prime \prime}\right) \rightarrow \cdots \rightarrow H_{0 p}^{\prime}\left(M_{* *}\right) \otimes_{K} L\left(\lambda^{\prime}-\lambda^{\prime \prime}\right) \\
& \rightarrow H_{q}\left(M_{*}^{\left(\lambda^{\prime \prime}\right)}\right) \otimes_{K} L\left(\lambda^{\prime}-\lambda^{\prime \prime}\right) \rightarrow 0
\end{aligned}
$$

is also an exact resolution by modules with VS by Proposition 4.17, and by Proposition 6.2 we obtain

$$
H_{p}^{\prime \prime}\left(H_{q p}^{\prime}\left(N_{* *}\right)\right) \cong W_{p}^{\lambda^{\prime}}\left[H_{q}\left(M_{*}^{\left(\lambda^{\prime \prime}\right)}\right) \otimes_{K} L\left(\lambda^{\prime}-\lambda^{\prime \prime}\right)\right] .
$$


But, again by Proposition 6.2, we obtain $H_{q}\left(M_{*}^{\left(\lambda^{\prime \prime}\right)}\right) \cong W_{q}^{\lambda^{\prime \prime}} M$. Thus, for the spectral sequence arising from the second filtration we have $E_{p q}^{2} \cong W_{p}^{\lambda^{\prime}}\left[W_{q}^{\lambda^{\prime \prime}} M \otimes_{K} L\left(\lambda^{\prime}-\lambda^{\prime \prime}\right)\right]$. Since $E_{p q}^{2} \underset{p}{\Rightarrow} H_{p+q}(\operatorname{Tot} N)=$ $T_{\lambda^{\prime \prime}, p+q}^{\lambda^{\prime}} M$, we have the result.

Corollary 6.4. For any $M \in \mathrm{Ob} C\left(\lambda^{\prime \prime}\right)$, we have

$$
T_{\lambda^{\prime \prime}}^{\lambda^{\prime}} M=W_{0}^{\lambda^{\prime}}\left[W_{0}^{\lambda^{\prime \prime}} M \otimes_{K} L\left(\lambda^{\prime}-\lambda^{\prime \prime}\right)\right] .
$$

7. An adjoint-like property of translation functors. We apply Corollary 6.4 to show that for certain nice modules in $C\left(\lambda^{\prime}\right)$ and $C\left(\lambda^{\prime \prime}\right)$, we have an adjoint-like property for the translation functors.

Definition 7.1. Let $\nu+\rho \in P^{+}$. We denote by $C(\nu)_{\text {nice }}$ the full subcategory of $C(\nu)$ whose objects are those modules $M \in \mathrm{Ob} C(\nu)$ such that every composition factor $L(\mu)$ of $M$ satisfies $w \cdot \mu \leq \nu$ for all $w \in W$.

REMARK. For the case $\nu=0$, this simply says that all composition factors are of the form $L(\mu)$ with $\mu$ in the Tits cone.

Lemma 7.2. Let $\nu+\rho \in P^{+}$. For any $M \in \mathrm{Ob} C(\nu)_{\text {nice }}$ and any $q=0,1, \ldots$, we have $W_{q}^{\nu} M \in \mathrm{Ob} C(\nu)_{\text {nice. }}$ Furthermore, if $q>0$, then every composition factor $L(\mu)$ of $W_{q}^{\nu} M$ satisfies $w \cdot \mu<\nu$ for all $w \in W$.

Proof. Consider a resolution

$$
\cdots \rightarrow M_{q} \rightarrow \cdots \rightarrow M_{0} \rightarrow M \rightarrow 0
$$

of $M$ by modules with VS in $C(\nu)$. By Proposition 6.2, we have that $W_{q}^{\nu} M=H_{q}\left(M_{*}^{(\nu)}\right)$, so that any composition factor of $W_{q}^{\nu} M$ is also a composition factor of $M_{q}^{(\nu)}$, and the first statement follows from Propositions 4.5 and 5.1. If $q>0$, then it was shown in [11, Theorem 6.9] that $W_{q}^{\nu} M$ has no composition factors of the form $L(w \cdot \nu)$, and the result follows.

LeMmA 7.3. Let $\nu+\rho \in P^{+}$. Suppose that $M$ is a module with an $R V S$ whose factors $M(\mu)$ each satisfy $w \cdot \mu \nless \nu$ for some $w \in W$, and suppose that $N \in \mathrm{Ob} C(\nu)_{\text {nice. }}$. Then there is a natural isomorphism $\operatorname{Hom}(M, N) \cong \operatorname{Hom}\left(M^{(\nu)}, N\right)$.

Proof. Our hypothesis on the Verma factors in the RVS means that the three step filtration of $M$ in Definition 5.2 is really just a two 
step filtration, so that we have $0=M_{0} \subseteq M_{1} \subseteq M_{2}=M_{3}=M$ with $M_{2} / M_{1}=M^{(\nu)}$ and where $M_{1}$ has an RVS whose factors $M(\mu)$ each satisfy $w \cdot \mu \not \leq \nu$ for some $w \in W$. The short exact sequence

$$
0 \rightarrow M_{1} \rightarrow M \rightarrow M^{(\nu)} \rightarrow 0
$$

gives a natural monomorphism $\phi: \operatorname{Hom}\left(M^{(\nu)}, N\right) \rightarrow \operatorname{Hom}(M, N)$, where $\phi$ is just composition with the natural projection $M \rightarrow M^{(\nu)}$. But consideration of the Verma factors of $M_{1}$ and the composition factors of $N$, together with Propositions 4.5 and 5.1 shows that $\operatorname{Hom}\left(M_{1}, N\right)=0$. Thus $\phi$ is an isomorphism.

Proposition 7.4. Let $\nu+\rho \in P^{+}$, let $V$ be a module with a finite $V S$ whose factors $M(\mu)$ are each of the form $M(w \cdot \nu)$ for some $w \in W$, and let $M \in \mathrm{Ob} C(\nu)_{\text {nice. }}$ Observe that $V \cong V^{(\nu)} \cong W_{0}^{\nu} V$. Then the natural map $\operatorname{Hom}(V, M) \rightarrow \operatorname{Hom}\left(V, W_{0}^{\nu} M\right)$ which sends $\phi$ to $W_{0}^{\nu} \phi$ is an isomorphism.

Proof. First, consider the case where $M \cong L(\mu)$ for some $\mu$ with $w \cdot \mu<\nu$ for all $w \in W$. Then, by Proposition 6.2 we have that $W_{0}^{\nu} L(\mu)=0$, so that $\operatorname{Ext}_{C(\nu)}^{n}\left(V, W_{0}^{\nu} L(\mu)\right)=0$ for all $n \geq 0$. On the other hand, by considering the Verma factors of $V$ and the hypothesis on $\mu$, Propositions 4.5, 4.6, and 5.1 imply that $\operatorname{Ext}_{C(\nu)}^{n}(V, L(\mu))=0$ for all $n \geq 0$.

Next, consider the case where $M \cong L(w \cdot \nu)$ for some $w \in W$. By Proposition 6.2, we have an epimorphism $\pi: W_{0}^{\nu} M \rightarrow M$ such that all composition factors of $X=\operatorname{Ker} \pi$ are of the form $L(\zeta)$ with $x \cdot \zeta<\nu$ for all $w \in W$. Thus, as in the above paragraph, we have $\operatorname{Ext}_{C(\nu)}^{n}(V, X)=0$ for all $n \geq 0$, and from the short exact sequence

$$
0 \rightarrow X \rightarrow W_{0}^{\nu} M \stackrel{\pi}{\rightarrow} M \rightarrow 0
$$

we obtain $\operatorname{Ext}_{C(\nu)}^{n}(V, M) \cong \operatorname{Ext}_{C(\nu)}^{n}\left(V, W_{0}^{\nu} M\right)$ for all $n \geq 0$, where the isomorphism is induced by $\pi$.

For the general case, let $0=V_{0} \subset V_{1} \subset \cdots \subset V_{n}=V$ be a VS for $V$, with factors $V_{i} / V_{i-1} \cong M\left(\lambda_{i}\right)$ for each $i=1, \ldots, n$, and choose $\mu \leq \lambda_{i}$ for all $i$. Let $0=M_{0} \subset M_{1} \subset \cdots \subset M_{k}=M$ be an LCS for $M$ at $\mu$. We use induction on $k$. For $k=1$, we are done in case $M_{1}$ is irreducible by the above paragraphs, and if $M_{1}$ has no weights $\geq \mu$, then neither does $W_{0}^{\nu} M_{1}$ by considering a resolution of $M_{1}$ by modules with VS as in Proposition 6.2, so that by Corollary 4.7 we have $\operatorname{Ext}\left(V, W_{0}^{\nu} M_{1}\right)=$ $0=\operatorname{Ext}\left(V, M_{1}\right)$ and $\operatorname{Hom}\left(V, W_{0}^{\nu} M_{1}\right)=0=\operatorname{Hom}\left(V, M_{1}\right)$. For $k>1$, 
from the short exact sequence

$$
0 \rightarrow M_{1} \rightarrow M \rightarrow M / M_{1} \rightarrow 0
$$

we obtain the commutative diagram with top row exact

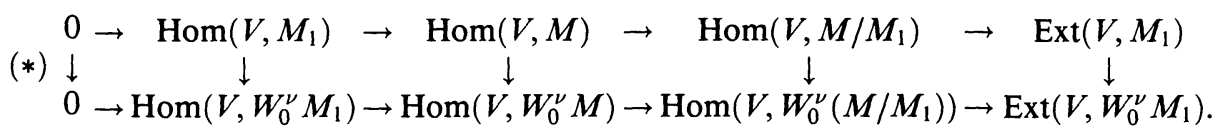

By the case $k=1$, the second and fifth vertical maps of $(*)$ are isomorphisms (which is why we did the extra work of studying Ext as well as Hom in the case $k=1$ ), and by the inductive hypothesis, the fourth vertical map of $(*)$ is an isomorphism. Of course, the first vertical map is also an isomorphism. Thus, if we can show that the bottom row is exact, then the Five Lemma will show that the third vertical map is an isomorphism, proving the result.

It remains to show that the bottom row of $(*)$ is exact. From the long exact homology sequence of Theorem 3.7, we have an exact sequence

$$
W_{1}^{\nu}\left(M / M_{1}\right) \stackrel{\phi}{\rightarrow} W_{0}^{\nu} M_{1} \rightarrow W_{0}^{\nu} M \rightarrow W_{0}^{\nu}\left(M / M_{1}\right) \rightarrow 0,
$$

and hence a short exact sequence

$$
0 \rightarrow\left(W_{0}^{\nu} M_{1}\right) / \operatorname{Im} \phi \rightarrow W_{0}^{\nu} M \rightarrow W_{0}^{\nu}\left(M / M_{1}\right) \rightarrow 0 .
$$

This gives the exactness of

$$
\begin{aligned}
0 & \rightarrow \operatorname{Hom}\left(V,\left(W_{0}^{\nu} M_{1}\right) / \operatorname{Im} \phi\right) \rightarrow \operatorname{Hom}\left(V, W_{0}^{\nu} M\right) \\
& \rightarrow \operatorname{Hom}\left(V, W_{0}^{\nu}\left(M / M_{1}\right)\right) \rightarrow \operatorname{Ext}\left(V,\left(W_{0}^{\nu} M_{1}\right) / \operatorname{Im} \phi\right) .
\end{aligned}
$$

Since $M \in \mathrm{Ob} C(\nu)_{\text {nice }}$, we have $M / M_{1} \in \mathrm{Ob} C(\nu)_{\text {nice }}$. Thus, by Lemma 7.2, every composition factor $L(\zeta)$ of $W_{1}^{\nu}\left(M / M_{1}\right)$ satisfies $w \cdot \zeta<\nu$ for all $w \in W$, and thus the same is true of $\operatorname{Im} \phi$. But this implies, by Propositions 4.5, 4.6, and 5.1, that $\operatorname{Ext}_{C(\nu)}^{n}(V, \operatorname{Im} \phi)=0$ for all $n \geq 0$, and hence $\operatorname{Ext}_{C(\nu)}^{n}\left(V,\left(W_{0}^{\nu} M_{1}\right) / \operatorname{Im} \phi\right) \cong \operatorname{Ext}_{C(\nu)}^{n}\left(V, W_{0}^{\nu} M_{1}\right)$ for all $n \geq 0$. Substituting this in $(* *)$ gives exactness of the bottom row of $(*)$.

COROLlary 7.5. Let $\nu+\rho \in P^{+}$, let $V$ be a module with $R V S$ whose factors $M(\zeta)$ each satisfy $w \cdot \zeta \nless \nu$ for some $w \in W$, and let $M \in$ $\mathrm{Ob} C(\nu)_{\text {nice. }}$. Then there is a natural isomorphism $\operatorname{Hom}\left(V^{(\nu)}, M\right) \cong$ $\operatorname{Hom}\left(V, W_{0}^{\nu} M\right)$.

Proof. By Proposition 7.4 and Lemma 7.3, we have natural isomorphisms $\operatorname{Hom}\left(V^{(\nu)}, M\right) \cong \operatorname{Hom}\left(V^{(\nu)}, W_{0}^{\nu} M\right) \cong \operatorname{Hom}\left(V, W_{0}^{\nu} M\right)$. 
Definition 7.6. For any g-module $M$, set $M^{(\mathfrak{h})}=\bigoplus_{\mu \in \mathfrak{h}^{*}} M_{\mu}$. For any weight module $M$, set ${ }^{*} M=\left(M^{*}\right)^{(\mathfrak{h})}$. Thus ${ }^{*} M=\left\{\phi \in M^{*} \mid \phi\right.$ vanishes on all but finitely many weight spaces of $M\}$, where the action on $M^{*}$ is given by $(x \cdot \phi)(m)=-\phi(x \cdot m)$ for all $m \in M, \phi \in M^{*}$, and $x \in \mathfrak{g}$. Note that ${ }^{*} L(\eta) \cong \Gamma(-\eta)$ and ${ }^{*} \Gamma(\eta) \cong L(-\eta)$ for any $\eta \in \mathfrak{h}^{*}$.

LemMA 7.7. Let $\mu, \nu \in \mathfrak{g}^{*}$, set $\eta=\mu-\nu$, and suppose $M \in \mathrm{Ob} C(\mu)$ and $N \in \mathrm{Ob} C(\nu)$. Then there is a natural isomorphism

$$
\operatorname{Hom}\left(M \otimes_{K} \Gamma(-\eta), N\right)=\operatorname{Hom}\left(M, L(\eta) \otimes_{K} N\right) .
$$

Proof. This of course is well known for a finite dimensional module in place of $L(\eta)$, and the only work that needs to be done is to check certain finiteness conditions in our present situation.

Let $\left\{n_{1}, n_{2}, \ldots\right\}$ be a $K$-basis for $N$ consisting of weight vectors. If $\phi \in \operatorname{Hom}\left(M \otimes_{K} \Gamma(-\eta), N\right)$ and $m \in M$, then there exists $\chi \in \mathfrak{h}^{*}$ with $m \in \sum_{\mu \geq \zeta \geq \chi} M_{\zeta}$, so that for any $v \in \Gamma(-\eta)$ we have $\phi(m \otimes v) \in \sum_{\nu \geq \zeta \geq \chi-\eta} N_{\zeta}$. Thus, using this $m \in M$, we may define linear functionals $\gamma_{1}, \gamma_{2}, \ldots$ on $\Gamma(-\eta)$ by $\phi(m \otimes v)=\sum_{i} \gamma_{i}(v) n_{i}$, and only finitely many of the $\gamma_{i}$ 's are nonzero. Note also that each $\gamma_{i}$ vanishes on all but finitely many weight spaces of $\Gamma(-\eta)$. To see this, if $v \in \Gamma(-\eta)_{\theta}$ then $\phi(m \otimes v) \in \sum_{\zeta \geq \chi} N_{\zeta+\theta}$ and $N_{\zeta+\theta} \neq 0$ implies $\chi+\theta \leq \zeta+\theta \leq \nu$, so that $\phi(m \otimes v) \neq 0$ implies $-\eta \leq \theta \leq \nu-\chi$. Thus we have that each $\gamma_{i} \in{ }^{*} \Gamma(-\eta)$. Identifying ${ }^{*} \Gamma(-\eta)$ with $L(\eta)$, we may define $\Phi(\phi)(m)=\sum_{i} \gamma_{i} \otimes n_{i} \in L(\eta) \otimes_{K} N$. One may easily check that this defines a linear map $\Phi: \operatorname{Hom}\left(M \otimes_{K} \Gamma(-\eta), N\right) \rightarrow \operatorname{Hom}\left(M, L(\eta) \otimes_{K} N\right)$.

Conversely, if $\psi \in \operatorname{Hom}\left(M, L(\eta) \otimes_{K} N\right)$ then for any $m \in M$, we may write $\psi(m)=\sum_{i} \gamma_{i} \otimes n_{i}$ and define $\Psi(\psi)(m \otimes v)=\sum_{i} \gamma_{i}(v) n_{i}$, where we identify $L(\eta)$ with ${ }^{*} \Gamma(-\eta)$, so that we obtain a linear map $\Psi: \operatorname{Hom}\left(M, L(\eta) \otimes_{K} N\right) \rightarrow \operatorname{Hom}\left(M \otimes_{K} \Gamma(-\eta), N\right)$.

It is routine to verify that $\Phi$ and $\Psi$ are inverses of each other.

We now resume the notation of $\S \S 5$ and 6 for the weights $\lambda^{\prime}$ and $\lambda^{\prime \prime}$.

Lemma 7.8. Let $M \in \mathrm{Ob} C\left(\lambda^{\prime \prime}\right)_{\text {nice. Then }}$

$$
M \otimes_{K} L\left(\lambda^{\prime}-\lambda^{\prime \prime}\right) \in \mathrm{Ob} C\left(\lambda^{\prime}\right)_{\text {nice }}
$$

Furthermore, if $M$ has no composition factors of the form $L\left(w \cdot \lambda^{\prime \prime}\right)$ with $w \in W$, then $M \otimes_{K} L\left(\lambda^{\prime}-\lambda^{\prime \prime}\right)$ has no composition factors of the form $L\left(w \cdot \lambda^{\prime}\right)$ with $w \in W$.

Proof. Suppose $L(\chi)$ is a composition factor of $M \otimes_{K} L\left(\lambda^{\prime}-\lambda^{\prime \prime}\right)$. Let $0=M_{0} \subset M_{1} \subset \cdots \subset M_{n}=M$ be an LCS for $M$ at $\chi+\lambda^{\prime \prime}-\lambda^{\prime}$. 
Then we have a filtration $0=M_{0} \otimes_{K} L\left(\lambda^{\prime}-\lambda^{\prime \prime}\right) \subset M_{1} \otimes_{K} L\left(\lambda^{\prime}-\lambda^{\prime \prime}\right) \subset$ $\cdots \subset M_{n} \otimes_{K} L\left(\lambda^{\prime}-\lambda^{\prime \prime}\right)=M \otimes_{K} L\left(\lambda^{\prime}-\lambda^{\prime \prime}\right)$, so that $L(\chi)$ must be a composition factor of some

$$
\begin{aligned}
\left(M_{i}\right. & \left.\otimes_{K} L\left(\lambda^{\prime}-\lambda^{\prime \prime}\right)\right) /\left(M_{i-1} \otimes_{K} L\left(\lambda^{\prime}-\lambda^{\prime \prime}\right)\right) \\
& \cong\left(M_{i} / M_{i-1}\right) \otimes_{K} L\left(\lambda^{\prime}-\lambda^{\prime \prime}\right),
\end{aligned}
$$

and since this can only happen in case $M_{i} / M_{i-1}$ has a weight $\geq \chi+$ $\lambda^{\prime \prime}-\lambda^{\prime}$, we see that $M_{i} / M_{i-1} \cong L(\mu)$ for some $\mu \geq \chi+\lambda^{\prime \prime}-\lambda^{\prime}$. Note that, since $M \in \mathrm{Ob} C\left(\lambda^{\prime \prime}\right)_{\text {nice }}$, we have $w \cdot \mu \leq \lambda^{\prime \prime}$ for all $w \in W$.

Now, since $L(\chi)$ is a composition factor of $L(\mu) \otimes_{K} L\left(\lambda^{\prime}-\lambda^{\prime \prime}\right)$, we see that $L(\chi)$ is also a composition factor of $M(\mu) \otimes_{K} L\left(\lambda^{\prime}-\lambda^{\prime \prime}\right)$. But the latter module has a VS by Proposition 4.17, so that $L(\chi)$ is a composition factor of one of the Verma factors $M(\zeta)$ in the VS. By Proposition 4.17, $\zeta$ is of the form $\mu+\nu$, where $\nu$ is a weight of $L\left(\lambda^{\prime}-\lambda^{\prime \prime}\right)$. Thus, for all $w \in W$, we have $w \cdot \zeta=w \cdot \mu+w \nu \leq$ $\lambda^{\prime \prime}+\left(\lambda^{\prime}-\lambda^{\prime \prime}\right)=\lambda^{\prime}$, with equality holding only if $w \cdot \mu=\lambda^{\prime \prime}$. Applying Propositions 4.5 and 5.1, we see that $w \cdot \chi \leq \lambda^{\prime}$ for all $w \in W$, and if equality holds then $w \cdot \mu=\lambda^{\prime \prime}$.

Lemma 7.9. Let $V \in \mathrm{Ob} C\left(\lambda^{\prime}\right)$ have an RVS whose factors $M(\zeta)$ each satisfy $w \cdot \zeta \nless \lambda^{\prime}$ for some $w \in W$. Then each factor $M(\zeta)$ in an RVS for $V \otimes_{K} \Gamma\left(\lambda^{\prime \prime}-\lambda^{\prime}\right)$ (which exists by Proposition 4.16) must satisfy $w \cdot \xi \nless \lambda^{\prime \prime}$ for some $w \in W$.

Proof. Each factor in an RVS for $V \otimes_{K} \Gamma\left(\lambda^{\prime \prime}-\lambda^{\prime}\right)$ is of the form $M(\xi)$ with $\xi=\zeta+\nu$, where $M(\zeta)$ is a Verma factor of $V$ and $\nu$ is a weight of $\Gamma\left(\lambda^{\prime \prime}-\lambda^{\prime}\right)$, by Proposition 4.16. Since $w \nu \geq \lambda^{\prime \prime}-\lambda^{\prime}$ for all $w \in W$, choosing $w$ so that $w \cdot \zeta \nless \lambda^{\prime}$ we obtain $w \cdot \xi=w \cdot \zeta+w \nu \nless \lambda^{\prime \prime}$.

We now have the tools necessary to prove the following adjointlike property relating the two translation functors.

THeOREM 7.10. Let $V \in \mathrm{Ob} C\left(\lambda^{\prime}\right)$ have a finite $V S$ whose factors $M(\zeta)$ each satisfy $w \cdot \zeta \nless \lambda^{\prime}$ for some $w \in W$, and let $M \in \mathrm{Ob} C\left(\lambda^{\prime \prime}\right)_{\text {nice. }}$. Then there is a natural isomorphism $\operatorname{Hom}\left(V, T_{\lambda^{\prime \prime}}^{\lambda^{\prime}} M\right) \cong \operatorname{Hom}\left(T_{\lambda^{\prime}}^{\lambda^{\prime \prime}} V, M\right)$.

Proof. We have

$$
\operatorname{Hom}\left(V, T_{\lambda^{\prime \prime}}^{\lambda^{\prime}} M\right) \cong \operatorname{Hom}\left(V, W_{0}^{\lambda^{\prime}}\left[W_{0}^{\lambda^{\prime \prime}} M \otimes_{K} L\left(\lambda^{\prime}-\lambda^{\prime \prime}\right)\right]\right)
$$

by Corollary 6.4. Since $W_{0}^{\lambda^{\prime \prime}} M \in \mathrm{Ob} C\left(\lambda^{\prime \prime}\right)_{\text {nice }}$ by Lemma 7.2, we have $W_{0}^{\lambda^{\prime \prime}} M \otimes_{K} L\left(\lambda^{\prime}-\lambda^{\prime \prime}\right) \in \mathrm{Ob} C\left(\lambda^{\prime}\right)_{\text {nice }}$ by Lemma 7.8. Thus, by Corollary 
7.5 ,

$$
\begin{aligned}
& \operatorname{Hom}\left(V, W_{0}^{\lambda^{\prime}}\left[W_{0}^{\lambda^{\prime \prime}} M \otimes_{K} L\left(\lambda^{\prime}-\lambda^{\prime \prime}\right)\right]\right) \\
& \quad \cong \operatorname{Hom}\left(V^{\left(\lambda^{\prime}\right)}, W^{\left(\lambda^{\prime}\right)}, W_{0}^{\lambda^{\prime \prime}} M \otimes_{K} L\left(\lambda^{\prime}-\lambda^{\prime \prime}\right)\right) .
\end{aligned}
$$

Also, by Lemma 7.7,

$$
\begin{aligned}
& \operatorname{Hom}\left(V^{\left(\lambda^{\prime}\right)}, W_{0}^{\lambda^{\prime \prime}} M \otimes_{K} L\left(\lambda^{\prime}-\lambda^{\prime \prime}\right)\right. \\
& \quad \cong \operatorname{Hom}\left(V^{\left(\lambda^{\prime}\right)} \otimes_{K} \Gamma\left(\lambda^{\prime \prime}-\lambda^{\prime}\right), W_{0}^{\lambda^{\prime \prime}} M\right) .
\end{aligned}
$$

By Lemma 7.9, an RVS for $V^{\left(\lambda^{\prime}\right)} \otimes_{K} \Gamma\left(\lambda^{\prime \prime}-\lambda^{\prime}\right)$ satisfies the hypotheses of Corollary 7.5, so that

$$
\begin{aligned}
& \operatorname{Hom}\left(V^{\left(\lambda^{\prime}\right)} \otimes_{K} \Gamma\left(\lambda^{\prime \prime}-\lambda^{\prime}\right), W_{0}^{\lambda^{\prime \prime}} M\right) \\
& \quad=\operatorname{Hom}\left(\left[V^{\left(\lambda^{\prime}\right)} \otimes_{K} \Gamma\left(\lambda^{\prime \prime}-\lambda^{\prime}\right)\right]^{\left(\lambda^{\prime \prime}\right)}, M\right) .
\end{aligned}
$$

But by Theorem 5.4, $\left[V^{\left(\lambda^{\prime}\right)} \otimes_{K} \Gamma\left(\lambda^{\prime \prime}-\lambda^{\prime}\right)\right]^{\left(\lambda^{\prime \prime}\right)} \cong T_{\lambda^{\prime}}^{\lambda^{\prime \prime}} V$.

8. Translation and Ext. We now apply the adjoint-like property of translation to compare Ext in various categories.

LEMMA 8.1. Let $\nu+\rho \in P^{+}$. Suppose $M \in \mathrm{Ob} C(\nu)$ has a VS whose factors $M(\mu)$ each satisfy $w \cdot \mu \nless \nu$ for some $w \in W$, and suppose $N \in$ $\mathrm{Ob} C(\nu)_{\text {nice. }}$ Then there is a natural isomorphism $\operatorname{Ext}_{C(\nu)}^{n}(M, N) \cong$ $\operatorname{Ext}_{C(\nu)_{\text {nice }}}^{n}\left(M^{(\nu)}, N\right)$ for each $n=0,1, \ldots$

Proof. By Propositions 3.11, 4.5, and 5.1, $M$ has a projective resolution in $C(\nu)$

$$
\cdots \rightarrow P_{n} \rightarrow \cdots \rightarrow P_{0} \rightarrow M \rightarrow 0
$$

in which each $P_{n}$ satisfies the hypotheses of Lemma 7.3, and in which all kernels and images also have finite VS satisfying the hypotheses of Lemma 7.3. Thus $\operatorname{Hom}\left(P_{n}, V\right)$ is naturally isomorphic to $\operatorname{Hom}\left(P_{n}^{(\nu)}, V\right)$ for any $V \in \mathrm{Ob} C(\nu)_{\text {nice, }}$, which shows that $P_{n}^{(\nu)}$ is projective in $C(\nu)_{\text {nice, }}$, and shows that the two complexes

$$
0 \rightarrow \operatorname{Hom}\left(P_{0}, N\right) \rightarrow \cdots \rightarrow \operatorname{Hom}\left(P_{n}, N\right) \rightarrow \cdots
$$

and

$$
0 \rightarrow \operatorname{Hom}\left(P_{0}^{(\nu)}, N\right) \rightarrow \cdots \rightarrow \operatorname{Hom}\left(P_{n}^{(\nu)}, N\right) \rightarrow \cdots
$$

are isomorphic. Since

$$
\cdots \rightarrow P_{n}^{(\nu)} \rightarrow \cdots \rightarrow P_{0}^{(\nu)} \rightarrow M^{(\nu)} \rightarrow 0
$$


is an exact projective resolution of $M^{(\nu)}$ in $C(\nu)_{\text {nice }}$ by Proposition 4.20 , we obtain

$$
\begin{aligned}
\operatorname{Ext}_{C(\nu)_{\text {nic }}}^{n}\left(M^{(\nu)}, N\right) & \cong H^{n}\left(\operatorname{Hom}\left(P_{*}^{(\nu)}, N\right)\right) \\
& \cong H^{n}\left(\operatorname{Hom}\left(P_{*}, N\right)\right) \cong \operatorname{Ext}_{C(\nu)}^{n}(M, N) .
\end{aligned}
$$

Lemma 8.2. Let $\nu \leq \lambda^{\prime}$ satisfy $w \cdot \nu \nless \lambda^{\prime}$ for some $w \in W$. Then $T_{\lambda^{\prime}}^{\lambda^{\prime \prime}} I^{\lambda^{\prime}}(\nu)$ is projective in $C\left(\nu^{\prime \prime}\right)_{\text {nice }}$.

Proof. The hypothesis on $\nu$ also holds for any $\mu$ such that $M(\mu)$ is a Verma factor of $I^{\lambda^{\prime}}(\nu)$, by Propositions 3.1, 4.5, and 5.1. Thus, for any $M \in \mathrm{Ob} C\left(\nu^{\prime \prime}\right)_{\text {nice }}$, we have a natural isomorphism $\operatorname{Hom}\left(\lambda_{\lambda^{\prime}}^{\lambda^{\prime \prime}} I^{\lambda^{\prime}}(\nu), M\right)$ $\cong \operatorname{Hom}\left(I_{\lambda}^{\lambda^{\prime}}(\nu), T_{\lambda^{\prime \prime}}^{\lambda^{\prime}} M\right)$ by Theorem 7.10. Now, if

$$
0 \rightarrow M^{\prime} \rightarrow M \rightarrow M^{\prime \prime} \rightarrow 0
$$

is a short exact sequence in $C\left(\lambda^{\prime \prime}\right)_{\text {nice, }}$, we have, by the right exactness of $T_{\lambda^{\prime \prime}}^{\lambda^{\prime}}$ and the projectivity of $I^{\lambda^{\prime}}(\nu)$, the exactness of

$$
\begin{aligned}
\operatorname{Hom}\left(I^{\lambda^{\prime}}(\nu), T_{\lambda^{\prime \prime}}^{\lambda^{\prime}} M^{\prime}\right) & \rightarrow \operatorname{Hom}\left(I^{\lambda^{\prime}}(\nu), T_{\lambda^{\prime \prime}}^{\lambda^{\prime}} M\right) \\
& \rightarrow \operatorname{Hom}\left(I^{\lambda^{\prime}}(\nu), T_{\lambda^{\prime \prime}}^{\lambda^{\prime}} M^{\prime \prime}\right) \rightarrow 0
\end{aligned}
$$

and hence, by the natural isomorphisms, the exactness of $\operatorname{Hom}\left(T_{\lambda^{\prime}}^{\lambda^{\prime \prime}}(\nu), M^{\prime}\right) \rightarrow \operatorname{Hom}\left(T_{\lambda^{\prime}}^{\lambda^{\prime \prime}} I^{\lambda^{\prime}}(\nu, M) \rightarrow \operatorname{Hom}\left(T_{\lambda^{\prime}}^{\lambda^{\prime \prime}} I^{\lambda^{\prime}}(\nu), M^{\prime \prime}\right) \rightarrow 0\right.$. This shows the right exactness of the functor $\operatorname{Hom}\left(T_{\lambda^{\prime}}^{\lambda^{\prime \prime}} I^{\lambda^{\prime}}(\nu),-\right)$ on $C\left(\lambda^{\prime \prime}\right)_{\text {nice }}$, so that $T_{\lambda^{\prime}}^{\lambda^{\prime \prime}} I^{\lambda^{\prime}}(\nu)$ is projective in $C\left(\lambda^{\prime \prime}\right)_{\text {nice }}$.

Theorem 8.3. Suppose $M \in \mathrm{Ob} C\left(\lambda^{\prime}\right)$ has a finite VS whose factors $M(\mu)$ each satisfy $w \cdot \mu \nless \lambda^{\prime}$ for some $w \in W$, and let $N \in \mathrm{Ob} C\left(\lambda^{\prime \prime}\right)_{\text {nice. }}$ Then

$$
\operatorname{Ext}_{C\left(\lambda^{\prime}\right)}^{n}\left(M, T_{\lambda^{\prime \prime}}^{\lambda^{\prime}} N\right) \cong \operatorname{Ext}_{C\left(\lambda^{\prime \prime}\right)}^{n}\left(T_{\lambda^{\prime}}^{\lambda^{\prime \prime}} M, N\right) \text { for all } n=0,1, \ldots
$$

Proof. By Propositions 3.11, 4.5 and 5.1, $M$ has an exact projective resolution in $C\left(\lambda^{\prime}\right)$

$$
\cdots \rightarrow P_{n} \rightarrow \cdots \rightarrow P_{0} \rightarrow M \rightarrow 0
$$

in which each $P_{n}$ is a finite direct sum of indecomposable projectives satisfying the hypotheses of Lemma 8.2, and in which all kernels and images have finite VS satisfying the same hypotheses as $M$. Thus, 
using the exactness asserted in Theorem 4.19 twice and the exactness of $-\otimes_{K} \Gamma\left(\lambda^{\prime \prime}-\lambda^{\prime}\right)$ once, we see that

$$
\cdots \rightarrow T_{\lambda^{\prime}}^{\lambda^{\prime \prime}} P_{n} \rightarrow \cdots \rightarrow T_{\lambda^{\prime}}^{\lambda^{\prime \prime}} P_{0} \rightarrow T_{\lambda^{\prime}}^{\lambda^{\prime \prime}} M \rightarrow 0
$$

is exact. But by Lemma 8.2, this is in fact a projective resolution of $T_{\lambda^{\prime}}^{\lambda^{\prime \prime}} M$ in $C\left(\lambda^{\prime \prime}\right)_{\text {nice }}$, so that $\operatorname{Ext}_{C\left(\lambda^{\prime \prime}\right)_{\text {nice }}}^{n}\left(T_{\lambda^{\prime}}^{\lambda^{\prime \prime}} M, N\right)$ is the $n$th cohomology of the complex

$$
0 \rightarrow \operatorname{Hom}\left(T_{\lambda^{\prime}}^{\lambda^{\prime \prime}} P_{0}, N\right) \rightarrow \cdots \rightarrow \operatorname{Hom}\left(T_{\lambda^{\prime}}^{\lambda^{\prime \prime}} P_{n}, N\right) \rightarrow \cdots .
$$

Since this complex is, by Theorem 7.10 , isomorphic to

$$
0 \rightarrow \operatorname{Hom}\left(P_{0}, T_{\lambda^{\prime \prime}}^{\lambda^{\prime}} N\right) \rightarrow \cdots \rightarrow \operatorname{Hom}\left(P_{n}, T_{\lambda^{\prime \prime}}^{\lambda^{\prime}} N\right) \rightarrow \cdots,
$$

and the $n$th cohomology of this latter complex is $\operatorname{Ext}_{C\left(\lambda^{\prime}\right)}^{n}\left(M, T_{\lambda^{\prime \prime}}^{\lambda^{\prime}} N\right)$, we have that $\operatorname{Ext}_{C\left(\lambda^{\prime \prime}\right) \text { mice }}^{n}\left(T_{\lambda^{\prime}}^{\lambda^{\prime \prime}} M, N\right) \cong \operatorname{Ext}_{C\left(\lambda^{\prime}\right)}^{n}\left(M, T_{\lambda^{\prime \prime}}^{\lambda^{\prime}} N\right)$. The result now follows from Lemma 8.1.

Lemma 8.4. Let $w \in W$. Then $\left(T_{\lambda^{\prime \prime}}^{\lambda^{\prime}} L\left(w \cdot \lambda^{\prime \prime}\right): L\left(w \cdot \lambda^{\prime}\right)\right)=1$ and all other composition factors $L(\mu)$ of $T_{\lambda^{\prime \prime}}^{\lambda^{\prime}} L\left(w \cdot \lambda^{\prime \prime}\right)$ satisfy $x \cdot \mu<\lambda^{\prime}$ for all $x \in W$.

Proof. We know that all composition factors $L(\mu)$ of $T_{\lambda^{\prime \prime}}^{\lambda^{\prime}} L\left(w \cdot \lambda^{\prime \prime}\right)$ satisfy $x \cdot \mu \leq \lambda^{\prime}$ for all $x \in W$, by applying Propositions 4.5 and 5.1 to a resolution of $L\left(w \cdot \lambda^{\prime \prime}\right)$ in $C\left(\lambda^{\prime \prime}\right)$ by modules with VS. Thus it suffices to show that for any $x \in W,\left(T_{\lambda^{\prime \prime}}^{\lambda^{\prime}} L\left(w \cdot \lambda^{\prime \prime}\right): L\left(x \cdot \lambda^{\prime}\right)\right)=\delta_{w, x}$. Recall that $T_{\lambda^{\prime \prime}}^{\lambda^{\prime}} L\left(w \cdot \lambda^{\prime \prime}\right) \cong W_{0}^{\lambda^{\prime}}\left[W_{0}^{\lambda^{\prime \prime}} L\left(w \cdot \lambda^{\prime \prime}\right) \otimes_{K} L\left(\lambda^{\prime}-\lambda^{\prime \prime}\right)\right]$ by Corollary 6.4 . Thus, by [11, Theorem 6.9],

$$
\left(T_{\lambda^{\prime \prime}}^{\lambda^{\prime}} L\left(w \cdot \lambda^{\prime \prime}\right): L\left(x \cdot \lambda^{\prime}\right)\right)=\left(W_{0}^{\lambda^{\prime \prime}} L\left(w \cdot \lambda^{\prime \prime}\right) \otimes_{K} L\left(\lambda^{\prime}-\lambda^{\prime \prime}\right): L\left(x \cdot \lambda^{\prime}\right)\right) .
$$

By Proposition 6.2, we have a short exact sequence

$$
0 \rightarrow M \rightarrow W_{0}^{\lambda^{\prime \prime}} L\left(w \cdot \lambda^{\prime \prime}\right) \rightarrow L\left(w \cdot \lambda^{\prime \prime}\right) \rightarrow 0
$$

in which every composition factor $L(\mu)$ of $M$ satisfies $y \cdot \mu<\lambda^{\prime \prime}$ for all $y \in W$. Since $-\otimes_{K} L\left(\lambda^{\prime}-\lambda^{\prime \prime}\right)$ is exact, we have a short exact sequence

$$
\begin{aligned}
0 & \rightarrow M \otimes_{K} L\left(\lambda^{\prime}-\lambda^{\prime \prime}\right) \rightarrow W_{0}^{\lambda^{\prime \prime}} L\left(w \cdot \lambda^{\prime \prime}\right) \otimes_{K} L\left(\lambda^{\prime}-\lambda^{\prime \prime}\right) \\
& \rightarrow L\left(w \cdot \lambda^{\prime \prime}\right) \otimes_{K} L\left(\lambda^{\prime}-\lambda^{\prime \prime}\right) \rightarrow 0,
\end{aligned}
$$

and by Lemma 7.8, $\left(M \otimes_{K} L\left(\lambda^{\prime}-\lambda^{\prime \prime}\right): L\left(x \cdot \lambda^{\prime}\right)\right)=0$. Thus we have

$$
\left(T_{\lambda^{\prime \prime}}^{\lambda^{\prime}} L\left(w \cdot \lambda^{\prime \prime}\right): L\left(x \cdot \lambda^{\prime}\right)\right)=\left(L\left(w \cdot \lambda^{\prime \prime}\right) \otimes_{K} L\left(\lambda^{\prime}-\lambda^{\prime \prime}\right): L\left(x \cdot \lambda^{\prime}\right)\right),
$$


and, using [11, Theorem 6.9] once again, we have

$$
\begin{aligned}
& \left(T_{\lambda^{\prime \prime}}^{\lambda^{\prime}} L\left(w \cdot \lambda^{\prime \prime}\right): L\left(x \cdot \lambda^{\prime}\right)\right) \\
& \quad=\left(W_{0}^{\lambda^{\prime}}\left[L\left(w \cdot \lambda^{\prime \prime}\right) \otimes_{K} L\left(\lambda^{\prime}-\lambda^{\prime \prime}\right)\right]: L\left(x \cdot \lambda^{\prime}\right)\right)=\delta_{w, x},
\end{aligned}
$$

where the last equality is the content of [11, Corollary 7.8].

REMARK. In [11], the translation functor $T_{\lambda^{\prime \prime}}^{\lambda^{\prime}}$ was defined in a slightly different way, so that $T_{\lambda^{\prime \prime}}^{\lambda^{\prime}} L\left(w \cdot \lambda^{\prime \prime}\right)$ was taken to be

$$
W_{0}^{\lambda^{\prime}}\left[L\left(w \cdot \lambda^{\prime \prime}\right) \otimes_{K} L\left(\lambda^{\prime}-\lambda^{\prime \prime}\right)\right]
$$

(cf. [11, Definition 7.1]). Thus, for the above application of [11, Corollary 7.8], the result in [11] states that $\left(T_{\lambda^{\prime \prime}}^{\lambda^{\prime}} L\left(w \cdot \lambda^{\prime \prime}\right): L\left(x \cdot \lambda^{\prime}\right)\right)=\delta_{w, x}$. Since we have used a different definition of the translation functor, we need a little extra work to obtain this result. The reason for our revision of the definition of the translation functors is that it gives us the adjoint-like properties of Theorems 7.10 and 8.3.

Proposition 8.5. Suppose $\lambda^{\prime}, \lambda^{\prime \prime} \in P^{+}$such that $\lambda^{\prime}-\lambda^{\prime \prime} \in P^{+}$, and let $x, y \in W$. Then

$$
\operatorname{Ext}_{C\left(\lambda^{\prime}\right)}^{n}\left(M\left(x \cdot \lambda^{\prime}\right), L\left(y \cdot \lambda^{\prime}\right)\right) \cong \operatorname{Ext}_{C\left(\lambda^{\prime \prime}\right)}^{n}\left(M\left(x \cdot \lambda^{\prime \prime}\right), L\left(y \cdot \lambda^{\prime \prime}\right)\right)
$$

for all $n=0,1, \ldots$

Proof. We have, using Proposition 5.9 and Theorem 8.3, that

$$
\begin{aligned}
& \operatorname{Ext}_{C\left(\lambda^{\prime \prime}\right)}^{n}\left(M\left(x \cdot \lambda^{\prime \prime}\right), L\left(y \cdot \lambda^{\prime \prime}\right)\right) \cong \operatorname{Ext}_{C\left(\lambda^{\prime \prime}\right)}^{n}\left(T_{\lambda^{\prime}}^{\lambda^{\prime \prime}} M\left(x \cdot \lambda^{\prime}\right), L\left(y \cdot \lambda^{\prime \prime}\right)\right) \\
& \quad \cong \operatorname{Ext}_{C\left(\lambda^{\prime}\right)}^{n}\left(M\left(x \cdot \lambda^{\prime}\right), T_{\lambda^{\prime \prime}}^{\lambda^{\prime}} L\left(y \cdot \lambda^{\prime \prime}\right)\right) .
\end{aligned}
$$

But, by Lemma 8.4, we may choose an LCS for $T_{\lambda^{\prime \prime}}^{\lambda^{\prime}} L\left(y \cdot \lambda^{\prime \prime}\right)$ at $x \cdot \lambda^{\prime}$, such that $L\left(y \cdot \lambda^{\prime}\right)$ occurs once and such that all other irreducible factors $L(\mu)$ satisfy $w \cdot \mu<\lambda^{\prime}$ for all $w \in W$. Thus, by Propositions 4.5, 4.6, and 5.1, we have that $\operatorname{Ext}_{C\left(\lambda^{\prime}\right)}^{m}\left(M\left(x \cdot \lambda^{\prime}\right), F\right)=0$ for any $m \geq 0$ for all factors $F$ other than $L\left(y \cdot \lambda^{\prime}\right)$ in the LCS, and hence

$$
\operatorname{Ext}_{C\left(\lambda^{\prime}\right)}^{n}\left(M\left(x \cdot \lambda^{\prime}\right), T_{\lambda^{\prime \prime}}^{\lambda^{\prime}} L\left(y \cdot \lambda^{\prime \prime}\right)\right) \cong \operatorname{Ext}_{C\left(\lambda^{\prime}\right)}^{n}\left(M\left(x \cdot \lambda^{\prime}\right), L\left(y \cdot \lambda^{\prime}\right)\right) .
$$

THeorem 8.6. Let $\lambda \in P^{+}$, and let $x, y \in W$. Then, for any $n=$ $0,1, \ldots, \operatorname{dim} \operatorname{Ext}_{C(\lambda)}^{n}(M(x \cdot \lambda), L(y \cdot \lambda))$ depends only on $x, y$, and $n$, but not on $\lambda$.

Proof. Since $0 \in P^{+}$and $\lambda-0 \in P^{+}$, we may apply the preceding proposition with $\lambda^{\prime}=\lambda$ and $\lambda^{\prime \prime}=0$ to obtain $\operatorname{Ext}_{C(\lambda)}^{n}(M(x \cdot \lambda), L(y \cdot \lambda)) \cong$ $\operatorname{Ext}_{C(0)}^{n}(M(x \cdot 0), L(y \cdot 0))$. 
REMARK. Similar results hold for other facettes in the closure of the dominant chamber. More precisely, if $\lambda^{\prime}+\rho, \lambda^{\prime \prime}+\rho \in P^{+}$with $\lambda^{\prime}-\lambda^{\prime \prime} \in$ $P^{+}$such that the stabilizers in $W$ of $\lambda^{\prime}$ and $\lambda^{\prime \prime}$ under the dot action are equal, then the proof of Proposition 8.5 carries over without change to show $\operatorname{Ext}_{C\left(\lambda^{\prime}\right)}^{n}\left(M\left(x \cdot \lambda^{\prime}\right), L\left(y \cdot \lambda^{\prime}\right)\right) \cong \operatorname{Ext}_{C\left(\lambda^{\prime \prime}\right)}^{n}\left(M\left(x \cdot \lambda^{\prime \prime}\right), L\left(y \cdot \lambda^{\prime \prime}\right)\right)$, and for Theorem 8.6 we replace 0 in the proof by a weight $\theta$ such that $\theta\left(h_{i}\right)=-1$ if $\lambda\left(h_{i}\right)=-1$ and $\theta\left(h_{i}\right)=0$ if $\lambda(h) \geq 0$.

9. Coherent continuation. We now specialize $\lambda^{\prime}$ and $\lambda^{\prime \prime}$ to the case where we are translating "to the $\alpha$-wall" and "from the $\alpha$-wall," for some simple root $\alpha$. These translations are used to define operators of coherent continuation, which were useful in proving the KazhdanLusztig conjecture for the case where $\mathfrak{g}$ is finite dimensional.

Throughout this section, we fix $\alpha=\alpha_{i}$ for some $i=1, \ldots, l$, fix $\lambda_{\alpha} \in P$ such that $\lambda_{\alpha}\left(h_{i}\right)=-1$ and $\lambda_{\alpha}\left(h_{j}\right) \geq 0$ for $j \neq i$, and fix $\lambda \in P^{+}$ such that $\lambda-\lambda_{\alpha} \in P^{+}$. Thus we have $\lambda$ in the dominant chamber and $\lambda_{\alpha}$ on the $\alpha$-wall of the dominant chamber. We denote the simple reflection $r_{i}$ by $r_{\alpha}$, as in $\S 2$. Observe that the stabilizers of $\lambda$ and $\lambda_{\alpha}$ in $W$ under the dot action are $W_{\lambda}=\{1\}$ and $W_{\lambda_{n}}=\left\{1, r_{\alpha}\right\}$. We apply the previous results with $\lambda^{\prime}=\lambda$ and $\lambda^{\prime \prime}=\lambda_{\alpha}$.

Definition 9.1. The operator of coherent continuation across the $\alpha$-wall is the functor $\Theta_{\alpha}$ from $C(\lambda)$ to $C(\lambda)$ defined by $\Theta_{\alpha}=T_{\lambda_{\alpha}}^{\lambda} \circ T_{\lambda}^{\lambda_{\alpha}}$.

Observe that $\Theta_{\alpha}$ is right exact, since both $T_{\lambda_{\alpha}}^{\lambda}$ and $T_{\lambda}^{\lambda_{\alpha}}$ are.

An immediate application of Theorem 8.3 to the present situation gives the following.

Proposition 9.2. Let $M \in \mathrm{Ob} C(\lambda)$ have a finite $V S$ whose factors $M(\mu)$ each satisfy $w \cdot \mu \nless \lambda$ for some $w \in W$, and let $N \in \mathrm{Ob} C(\lambda)$. Then for all $n \geq 0$, we have $\operatorname{Ext}_{C(\lambda)}^{n}\left(M, \Theta_{\alpha} N\right) \cong \operatorname{Ext}_{C\left(\lambda_{n}\right)}^{n}\left(T_{\lambda}^{\lambda_{\mu}} M, T_{\lambda}^{\lambda_{n}} N\right)$.

Definition 9.3. The Bruhat order on $W$ is defined by $w_{1} \leq w_{2}$ if there exist $\beta_{1}, \ldots, \beta_{n} \in \Delta_{R}^{+}$such that $l\left(w_{1}\right) \leq l\left(w_{1} r_{\beta_{1}}\right) \leq \cdots \leq$ $l\left(w_{1} r_{\beta_{1}} \cdots r_{\beta_{n}}\right)$ and $w_{1} r_{\beta_{1}} \ldots r_{\beta_{n}}=w_{2}$.

LeMma 9.4. Let $w \in W$ such that $w<w r_{\alpha}$. Then there is a short exact sequence

$$
0 \rightarrow M(w \cdot \lambda) \rightarrow T_{\lambda_{\alpha}}^{\lambda} M\left(w \cdot \lambda_{\alpha}\right) \rightarrow M\left(\left(w r_{\alpha}\right) \cdot \lambda\right) \rightarrow 0 .
$$

Proof. By Proposition 5.8, $T_{\lambda_{\alpha}}^{\lambda} M\left(w \cdot \lambda_{\alpha}\right)$ has a VS with two factors, $M(w \cdot \lambda)$ and $M\left(\left(w r_{\alpha}\right) \cdot \lambda\right)$, each with multiplicity one. The result now 
follows from the fact that $\operatorname{Ext}\left(M(w \cdot \lambda), M\left(\left(w r_{\alpha}\right) \cdot \lambda\right)\right)=0$ by Corollary 4.7.

LEMMA 9.5. Let $w \in W$ such that $w<w r_{\alpha}$. Then $T_{\lambda}^{\lambda_{n}} M(w \cdot \lambda) \cong$ $M\left(w \cdot \lambda_{\alpha}\right) \cong T_{\lambda}^{\lambda^{\lambda_{1}}} M\left(\left(w r_{\alpha}\right) \cdot \lambda\right)$. Furthermore, if we choose an imbedding $\phi: M\left(\left(w r_{\alpha}\right) \cdot \lambda\right) \rightarrow M(w \cdot \lambda)$, then $T_{\lambda}^{\lambda_{\text {w }}} \phi$ is an isomorphism.

Proof. By Proposition 5.9,

$$
T_{\lambda}^{\lambda_{n}} M(w \cdot \lambda) \cong M\left(w \cdot \lambda_{\alpha}\right) \text { and } T_{\lambda}^{\lambda_{n}} M\left(\left(w r_{\alpha}\right) \cdot \lambda\right) \cong M\left(\left(w r_{\alpha}\right) \cdot \lambda_{\alpha}\right) \text {. }
$$

But $r_{\alpha} \cdot \lambda_{\alpha}=\lambda_{\alpha}$, so that $M\left(\left(w r_{\alpha}\right) \cdot \lambda_{\alpha}\right)=M\left(w \cdot \lambda_{\alpha}\right)$, and we have the first assertion. For the second assertion, since any nonzero map between Verma modules is injective, it suffices to show that $T_{\lambda}^{\lambda_{a}} \phi$ is not the zero map. But this map induces

$$
\begin{aligned}
\left(T_{\lambda}^{\lambda_{n}} \phi\right)^{*} & : \operatorname{Hom}\left(T_{\lambda}^{\lambda_{n}} M(w \cdot \lambda), M\left(w \cdot \lambda_{\alpha}\right)\right) \\
& \rightarrow \operatorname{Hom}\left(T_{\lambda}^{\lambda_{n}} M\left(\left(w r_{\alpha}\right) \cdot \lambda\right), M\left(w \cdot \lambda_{\alpha}\right)\right),
\end{aligned}
$$

and by the natural isomorphism of Theorem 7.10, we have the commutative square

$$
\begin{array}{ccc}
\operatorname{Hom}\left(T_{\lambda}^{\lambda_{n}} M(w \cdot \lambda), M\left(w \cdot \lambda_{\alpha}\right)\right) & \stackrel{\left(T_{\left.\lambda_{n} \phi\right)^{*}}\right.}{\rightarrow} & \operatorname{Hom}\left(T_{\lambda}^{\lambda_{\mu}} M\left(\left(w r_{\alpha}\right) \cdot \lambda\right), M\left(w \cdot \lambda_{\alpha}\right)\right) \\
\downarrow & \downarrow & \downarrow \\
\operatorname{Hom}\left(M(w \cdot \lambda), T_{\lambda_{\alpha}}^{\lambda} M\left(w \cdot \lambda_{\alpha}\right)\right) & \stackrel{\phi^{*}}{\rightarrow} & \operatorname{Hom}\left(M\left(\left(w r_{\alpha}\right) \cdot \lambda\right), T_{\lambda_{\mu}}^{\lambda} M\left(w \cdot \lambda_{\alpha}\right)\right),
\end{array}
$$

where the bottom map is the restriction map, which is nonzero in view of Lemma 9.4. Thus $\left(T_{\lambda}^{\lambda_{n}} \phi\right)^{*} \neq 0$, so that $T_{\lambda}^{\lambda_{n}} \phi \neq 0$.

We close this section with a summary of several properties of the operators of coherent continuation.

Proposition 9.6. Suppose $w \in W$ such that $w<w r_{\alpha}$. Then

(i) Any imbedding $\phi: M\left(\left(w r_{\alpha}\right) \cdot \lambda\right) \rightarrow M(w \cdot \lambda)$ induces an isomorphism $\Theta_{\alpha} \phi: \Theta_{\alpha} M\left(\left(w r_{\alpha}\right) \cdot \lambda\right) \rightarrow \Theta_{\alpha} M(w \cdot \lambda)$.

(ii) $\Theta_{\alpha} L(w \cdot \lambda)=0$.

(iii) There is a short exact sequence

$$
0 \rightarrow M(w \cdot \lambda) \rightarrow \Theta_{\alpha} M(w \cdot \lambda) \rightarrow M\left(\left(w r_{\alpha}\right) \cdot \lambda\right) \rightarrow 0
$$

which does not split.

(iv) $\Theta_{\alpha} L\left(\left(w r_{\alpha}\right) \cdot \lambda\right) \neq 0$.

Proof. By Lemmas 9.4 and 9.5, together with the definition of $\Theta_{\alpha}$, we obtain (i) and the existence of the short exact sequence in (iii). If 
the sequence were split, we would have

$$
\begin{aligned}
\operatorname{dim} \operatorname{Hom}\left(M\left(\left(w r_{\alpha}\right) \cdot \lambda\right), \Theta_{\alpha} M(w \cdot \lambda)\right) \\
=\operatorname{dim} \operatorname{Hom}\left(M\left(\left(w r_{\alpha}\right) \cdot \lambda\right), M\left(\left(w r_{\alpha}\right) \cdot \lambda\right)\right) \\
\quad+\operatorname{dim} \operatorname{Hom}\left(M\left(\left(w r_{\alpha}\right) \cdot \lambda\right), M(w \cdot \lambda)\right)=2 .
\end{aligned}
$$

But, by Proposition 9.2,

$$
\begin{aligned}
\operatorname{dim} & \operatorname{Hom}\left(M\left(\left(w r_{\alpha}\right) \cdot \lambda\right), \Theta_{\alpha} M(w \cdot \lambda)\right) \\
& =\operatorname{dim} \operatorname{Hom}\left(T_{\lambda}^{\lambda_{\alpha}} M\left(\left(w r_{\alpha}\right) \cdot \lambda\right), T_{\lambda}^{\lambda_{\text {se }}} M(w \cdot \lambda)\right) \\
& =\operatorname{dim} \operatorname{Hom}\left(M\left(w \cdot \lambda_{\alpha}\right), M\left(w \cdot \lambda_{\alpha}\right)\right)=1 .
\end{aligned}
$$

Thus, the sequence does not split.

Now, fix an imbedding $\phi: M\left(\left(w r_{\alpha}\right) \cdot \lambda\right) \rightarrow M(w \cdot \lambda)$, and let $X=$ Coker $\phi$. By the right exactness of $\Theta_{\alpha}$, we have the exact sequence

$$
\Theta_{\alpha} M\left(\left(w r_{\alpha}\right) \cdot \lambda\right) \stackrel{\Theta_{\alpha} \phi}{\rightarrow} \Theta_{\alpha} M(w \cdot \lambda) \rightarrow \Theta_{\alpha} X \rightarrow 0 .
$$

But $\Theta_{\alpha} \phi$ is an isomorphism, by (i), so that $\Theta_{\alpha} X=0$. Since $L(w \cdot \lambda)$ is a quotient of $X$, (ii) now follows from the right exactness of $\Theta_{\alpha}$.

To prove (iv), it suffices to show that

$$
\operatorname{Hom}\left(M(w \cdot \lambda), \Theta_{\alpha} L\left(\left(w r_{\alpha}\right) \cdot \lambda\right)\right)
$$

is nonzero, and hence, by Proposition 9.2 and Lemma 9.5, that $\operatorname{Hom}\left(M\left(w \cdot \lambda_{\alpha}\right), T_{\lambda}^{\lambda_{\alpha}} L\left(\left(w r_{\alpha}\right) \cdot \lambda\right)\right) \neq 0$. Let $M$ be the unique maximal submodule of $M\left(\left(w r_{\alpha}\right) \cdot \lambda\right)$, so that we have the short exact sequence

$$
0 \rightarrow M \rightarrow M\left(\left(w r_{\alpha}\right) \cdot \lambda\right) \rightarrow L\left(\left(w r_{\alpha}\right) \cdot \lambda\right) \rightarrow 0,
$$

and let

$$
\cdots \rightarrow M_{n} \rightarrow \cdots \rightarrow M_{1} \rightarrow M \rightarrow 0
$$

be the exact resolution of $M$ in $C(\lambda)$ obtained by tensoring $M$ over $K$ with the standard resolution of the trivial module. Thus, for each $n=1,2, \ldots$ we have $M_{n}=\left(U(\mathfrak{g}) \otimes_{U(\mathfrak{b})} \bigwedge^{n-1}(\mathfrak{g} / \mathfrak{b})\right) \otimes_{K} M$, so that each $M_{n}$ has a VS whose factors $M(\mu)$ each satisfy $\mu<\left(w r_{\alpha}\right) \cdot \lambda$. Splicing the sequences, we obtain an exact resolution

$$
\cdots \rightarrow M_{n} \rightarrow \cdots \rightarrow M_{1} \rightarrow M_{0} \rightarrow L\left(\left(w r_{\alpha}\right) \cdot \lambda\right) \rightarrow 0
$$

of $L\left(\left(w r_{\alpha}\right) \cdot \lambda\right)$ in $C(\lambda)$, where $M_{0}=M\left(\left(w r_{\alpha}\right) \cdot \lambda\right)$. Note that this resolution satisfies the hypotheses of Corollary 5.6. Thus $T_{\lambda}^{\lambda_{n}} L\left(\left(w r_{\alpha}\right) \cdot \lambda\right)$ is the 0 th homology of the complex

$$
\cdots \rightarrow T_{\lambda}^{\lambda_{n}} M_{n} \rightarrow \cdots \rightarrow T_{\lambda}^{\lambda_{\lambda_{n}}} M_{1} \rightarrow T_{\lambda}^{\lambda_{n}} M_{0} \rightarrow 0 .
$$


Now $T_{\lambda}^{\lambda_{n}} M_{0}=T_{\lambda}^{\lambda_{n}} M\left(\left(w r_{\alpha}\right) \cdot \lambda\right) \cong M\left(w \cdot \lambda_{\alpha}\right)$ by Lemma 9.5, and $M_{1}$ has a VS in which any factor of the form $M(x \cdot \lambda)$ must satisfy $x \cdot \lambda<\left(w r_{\alpha}\right) \cdot \lambda<w \cdot \lambda$, so that the VS for $T_{\lambda}^{\lambda_{\mu}} M_{1}$, all factors are of the form $M\left(x \cdot \lambda_{\alpha}\right)$ with $x \cdot \lambda_{\alpha}<w \cdot \lambda_{\alpha}$. Thus we see that $T_{\lambda}^{\lambda_{\alpha}} L\left(\left(w r_{\alpha}\right) \cdot \lambda\right) \cong$

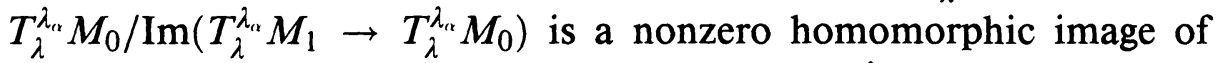
$M\left(w \cdot \lambda_{\alpha}\right)$. This shows that $\operatorname{Hom}\left(M\left(w \cdot \lambda_{\alpha}\right), T_{\lambda}^{\lambda_{u}} L\left(\left(w r_{\alpha}\right) \cdot \lambda\right)\right) \neq 0$, proving (iv).

10. Kazhdan-Lusztig polynomials. We define certain polynomials $\bar{P}_{x, y}$ for each $x, y \in W$, which are known in the finite dimensional case to be similar to those introduced in [8], the difference being that we work with a dominant weight rather than an antidominant weight. Throughout this section we fix $\lambda \in P^{+}$.

Definition 10.1. Let $x, y \in W$, and let $t$ be an indeterminate. Define a polynomial $\bar{P}_{x, y}(t) \in \mathbb{Z}[t]$ by

$$
\bar{P}_{x, y}(t)=\sum_{k \geq 0} \operatorname{dim} \operatorname{Ext}_{C(\lambda)}^{k}(M(x \cdot \lambda), L(y \cdot \lambda)) t^{l(x)-l(y)_{k}} .
$$

Remarks. By Propositions 4.5, 4.6, and 5.1, we have that

$$
\operatorname{Ext}_{C(\lambda)}^{k}(M(x \cdot \lambda), L(y \cdot \lambda))=0
$$

unless $l(x)-l(y) \geq k$, so that $\bar{P}_{x, y}(t)$ is indeed a polynomial in $\mathbb{Z}[t]$. Also, by Theorem 8.6, the polynomial $\bar{P}_{x, y}$ depends only on $x$ and $y$, not on the choice of $\lambda \in P^{+}$. For the case where $\mathfrak{g}$ is finite dimensional, if we set $q=t^{2}$ and view $\bar{P}_{x, y}$ as an element of $\mathbb{Z}\left[q^{1 / 2}\right]$, it is shown in [15, Theorem 7.3] that $\bar{P}_{x, y}$ is the Kazhdan-Lusztig polynomial $P_{x w_{0}, y w_{0}}$, where $w_{0}$ is the longest element of $W$. The reason for the appearance of $w_{0}$ is that we are taking $\lambda$ to be dominant rather than antidominant.

Definition 10.2. For any $M \in \mathrm{Ob} C(\lambda)$, and any $\mu \leq \lambda$, define $[M: M(\mu)] \in \mathbb{Z}$ to be the coefficient $a_{\mu}$ in the expression $\operatorname{ch} M=$ $\sum_{\mu \leq \lambda} a_{\mu} \operatorname{ch} M(\mu)$.

REMARK. Such an expression for $\operatorname{ch} M$ exists, by taking an exact resolution of $M$ in $C(\lambda)$ by modules with VS. It is unique by [10, Lemma 5.3].

For the next proposition, recall the integers $g_{i}(\nu, \mu)$ which were introduced in Definition 3.10. 
Proposition 10.3. For any $x, y \in W$,

$$
[L(y \cdot \lambda): M(x \cdot \lambda)]=\sum_{k \geq 0}(-1)^{k} \operatorname{dim} \operatorname{Ext}_{C(\lambda)}^{k}(M(x \cdot \lambda), L(y \cdot \lambda)) .
$$

Proof. Consider the projective resolution of $M(x \cdot \lambda)$ in $C(\lambda)$

$$
\cdots \rightarrow P_{k} \rightarrow \cdots \rightarrow P_{0} \rightarrow M(x \cdot \lambda) \rightarrow 0
$$

given by Proposition 3.11, so that each $P_{k} \cong \bigoplus_{\nu \leq \lambda} g_{k}(\nu, x \cdot \lambda) I^{\lambda}(\nu)$. Then for each $k=0,1, \ldots$ we have

$$
\begin{aligned}
\operatorname{dim} \operatorname{Hom}\left(P_{k}, L(y \cdot \lambda)\right) & =\sum_{\nu \leq \lambda} g_{k}(\nu, x \cdot \lambda) \operatorname{dim} \operatorname{Hom}\left(I^{\lambda}(\nu), L(y \cdot \lambda)\right) \\
& =g_{k}(y \cdot \lambda, x \cdot \lambda),
\end{aligned}
$$

by Proposition 3.1. The Euler-Poincare principle gives

$$
\begin{aligned}
\sum_{k \geq 0}(-1)^{k} & \operatorname{dim} \operatorname{Ext}_{C(\lambda)}^{k}(M(x \cdot \lambda), L(y \cdot \lambda)) \\
& =\sum_{k \geq 0}(-1)^{k} \operatorname{dim} H^{k}\left(\operatorname{Hom}\left(P_{*}, L(y \cdot \lambda)\right)\right) \\
& =\sum_{k \geq 0}(-1)^{k} \operatorname{dim} \operatorname{Hom}\left(P_{k}, L(y \cdot \lambda)\right)=\sum_{k \geq 0}(-1)^{k} g_{k}(y \cdot \lambda, x \cdot \lambda) .
\end{aligned}
$$

Now, it was shown in [10, Lemma 5.2], using a Möbius inversion argument, that

$$
[L(y \cdot \lambda): M(x \cdot \lambda)]=\sum_{k \geq 0}(-1)^{k} g_{k}(y \cdot \lambda, x \cdot \lambda),
$$

and the result follows.

Corollary 10.4. For any $x, y \in W$,

$$
[L(y \cdot \lambda): M(x \cdot \lambda)]=(-1)^{l(x)-l(y)} \bar{P}_{x, y}(-1) .
$$

Conjecture 10.5. $\operatorname{Ext}_{C(\lambda)}^{k}(M(x \cdot \lambda), L(y \cdot \lambda))=0$ unless $l(x)-l(y)=k$ $(\bmod 2)$.

This conjecture was shown to hold in the finite dimensional case in [1], [2], and [15], along with the Kazhdan-Lusztig conjecture.

COROllary 10.6. If Conjecture 10.5 holds, then, letting $q=t^{2}$ so that we view $\bar{P}_{x, y}$ as $\bar{P}_{x, y}(q) \in \mathbb{Z}\left[q^{1 / 2}\right]$, we have for any $x, y \in W$ that $[L(y \cdot \lambda): M(x \cdot \lambda)]=(-1)^{l(x)-l(y)} \bar{p}_{x, y}(1)$.

What we would like to have is a combinatorial algorithm for computing the polynomials $\bar{P}_{x, y}$ similar to the algorithm in [8], where the 
Kazhdan-Lusztig polynomials were introduced. We are missing several tools which are available in the finite dimensional case, however. Among these is a stronger version of Proposition 9.2 which allows moving the operators $\Theta_{\alpha}$ back and forth between the two arguments to the Ext bifunctor. We nevertheless summarize here certain properties of the polynomials $\bar{P}_{x, y}$.

TheOREM 10.7. Let $x, y \in W$. Then

(i) $\operatorname{deg}_{t} \bar{P}_{x, y}(t) \leq l(x)-l(y)$.

(ii) $\bar{P}_{x, y}=0$ unless $x \geq y$.

(iii) $\bar{P}_{x, x}=1$ for any $x \in W$.

(iv) $\bar{P}_{1, x}=\delta_{1, x}$ (Kronecker symbol).

(v) $\bar{P}_{x, 1}=1$ for all $x \in W$.

Proof. By Propositions 4.5, 4.6, and 5.1, if $\operatorname{Ext}_{C(\lambda)}^{n}(M(x \cdot \lambda), L(y \cdot \lambda))$ is nonzero, then there exist $w_{0}, w_{1}, \ldots, w_{n} \in W$ such that $x \cdot \lambda=$ $w_{0} \cdot \lambda \leq w_{1} \cdot \lambda \leq \cdots \leq w_{n} \cdot \lambda=y \cdot \lambda$ and such that $M\left(w_{i-1} \cdot \lambda\right)$ imbeds in $M\left(w_{i} \cdot \lambda\right)$ for each $i=1, \ldots, n$. But then $x=w_{0}>w_{1}>\cdots>w_{n}=y$ in the Bruhat order by [12, Theorem 4.3]. The first four assertions follow from this fact and the definition of the polynomials $\bar{P}_{x, y}$. For the fifth assertion, by Theorem 8.6 we may assume that $\lambda=0$, so that $1 \cdot \lambda=\lambda=0$ and $L(\lambda)=L(0)$ is the trivial module. Now, it was shown in [3, Theorem 2] that for each $n \geq 0, \operatorname{Ext}_{C(0)}^{n}(M(x \cdot 0), L(0)) \cong$ $H^{n}\left(\mathfrak{n}^{+}, L(0)\right)_{x \cdot 0}$. Recall the standard complex

$$
0 \rightarrow C_{0} \rightarrow C_{1} \rightarrow \cdots \rightarrow C_{n} \rightarrow \cdots
$$

used to compute the $\mathfrak{n}^{+}$-cohcmology of $L(0)$, where each $C_{n}=$ $\operatorname{Hom}_{K}\left(\bigwedge^{n} \mathfrak{n}^{+}, L(0)\right)$ and the cohomology $\delta: C_{n} \rightarrow C_{n+1}$ is defined by

$$
\begin{aligned}
(\delta c)\left(x_{1} \cdots x_{n+1}\right)= & \sum_{i=1}^{n+1}(-1)^{i+1} x_{i} \cdot c\left(x_{1} \wedge \ldots \hat{x}_{i} \cdots \wedge x_{n+1}\right) \\
& +\sum_{i<j}(-1)^{i+j} c\left(\left[x_{i}, x_{j}\right] \wedge x_{1} \wedge \cdots \hat{x}_{i} \cdots \hat{x}_{j} \cdots \wedge x_{n+1}\right)
\end{aligned}
$$

for all $c \in C_{n}$ and all $x_{1}, \ldots, x_{n+1} \in \mathfrak{n}^{+}$. Since $\delta$ is an $\mathfrak{h}$-module homomorphism, $H^{n}\left(\mathfrak{n}^{+}, L(0)\right)_{x \cdot 0}$ may be computed as the $n$th cohomology of the restriction of the standard complex to the $x \cdot 0$-weight spaces

$$
0 \rightarrow\left(C_{0}\right)_{x \cdot 0} \rightarrow\left(C_{1}\right)_{x \cdot 0} \rightarrow \cdots
$$

But $\operatorname{dim}\left(C_{n}\right)_{x \cdot 0}=\operatorname{dim} \operatorname{Hom}_{K}\left(\bigwedge^{n} \mathfrak{n}^{+}, L(0)\right)_{x \rho-\rho}=\operatorname{dim}\left(\bigwedge^{n} \mathfrak{n}^{+}\right)_{\rho-x \rho}$, so that $\operatorname{dim}\left(C_{n}\right)_{x \cdot 0}$ is equal to the number of partitions of $\rho-x \rho$ into a 
sum of $n$ distinct positive roots (with their appropriate multiplicities). It was shown in [5, Proposition 2.4] that the only set of positive roots which has a sum equal to $\rho-x \rho$ is the set $\Phi_{x}=\left\{\beta \in \Delta^{+} \mid x^{-1} \beta<\right.$ $0\}$, and this set has cardinality $l(x)$, by [5, Proposition 2.2]. Thus $\operatorname{dim}\left(C_{n}\right)_{x \cdot 0}=\delta_{l(x), n}$, and the restrictions of $\delta$ to the $x \cdot 0$ weight spaces are all the zero map, so that $\operatorname{dim} H^{n}\left(\mathfrak{n}^{+}, L(0)\right)_{x \cdot 0}=\delta_{l(x), n}$, and (v) follows from the definition of the polynomial $\bar{P}_{x, 1}$.

REMARK. Since so little is known about the multiplicities

$$
(M(y \cdot \lambda): L(x \cdot \lambda)) \text { and }[L(y \cdot \lambda): M(x \cdot \lambda)] \text {, }
$$

or about the dimensions $\operatorname{dim} \operatorname{Ext}^{n}(M(x \cdot \lambda), L(y \cdot \lambda))$, in the case of a nonsymmetrizable GCM, due in large part to the difficulty of computing the character of $M(x \cdot \lambda)$, it is difficult to compute examples of the polynomials $\bar{P}_{x, y}(t)$. However, one can show, using Proposition 10.3, that when $x>y$ with $l(x)-l(y)=1$, we have the constant polynomial

$$
\bar{P}_{x, y}(t)=(M(y \cdot \lambda): L(x \cdot \lambda))=-[L(y \cdot \lambda): M(x \cdot \lambda)] .
$$

It would be interesting to be able to compute examples of $\bar{P}_{x, y}(t)$ with $l(x)-l(y) \geq 2$ to see if they match the corresponding Kazhdan-Lusztig polynomials.

Acknowledgment. The author wishes to thank the referee for several helpful suggestions for improving the exposition. These comments were greatly appreciated.

\section{REFERENCES}

[1] A Beilinson and J. Bernstein, Localisation de $\mathfrak{g}$-modules, C. R. Acad. Sci. Paris, 292 (1981), 15-18.

[2] J. L. Brylinski and M. Kashiwara, Démonstration de la conjecture de KazhdanLusztig sur les modules de Verma, C. R. Acad. Sci. Paris, 291 (1980), 373-376.

[3] P. Delorme, Extensions in the category $\mathcal{O}$ of Bernstein-Gelfand-Gelfand, applications, (Russian), Funkt. Anal. i Ego Prilozhenia, 14:3 (1980), 77-78; (English), Functional Anal. Appl., 14 (1980), 228-229.

[4] V. V. Deodhar, O. Gabber and V. G. Kac, Structure of some categories of representations of infinite-dimensional Lie algebras, Adv. in Math., 45 (1982), 92116.

[5] H. Garland and J. Lepowsky, Lie algebra homology and the Macdonald-Kac formulas, Invent. Math., 34 (1976), 37-76.

[6] J. C. Jantzen, Moduln mit einem Höchsten Gewicht, Springer-Verlag, Lecture Notes in Mathematics, 750, Berlin (1979).

[7] V. G. Kac, Simple irreducible graded Lie algebras of finite growth, (Russian), Izv. Akad. Nauk SSSR, 32 (1968), 1323-1367; (English), Math. USSR-Izv., 2 (1968), 1271-1311. 
[8] D. Kazhdan and G. Lusztig, Representations of Coxeter groups and Hecke algebras, Invent. Math., 53 (1979), 165-184.

[9] R. V. Moody, A new class of Lie algebras, J. Algebra, 10 (1968), 211-230.

[10] W. Neidhardt, The BGG resolution, character and denominator formulas, and related results for Kac-Moody algebras, Trans. Amer. Math. Soc., 297 (1986), 487-504.

[11] W. Neidhardt, Local projective resolutions and translation functors for KacMoody algebras, Trans. Amer. Math. Soc., 305 (1988), 221-245.

[12] W. Neidhardt, Verma module imbeddings and the Bruhat order for Kac-Moody algebras, J. Algebra, 109 (1987), 430-438.

[13] A. Rocha-Caridi and N. Wallach, Projective modules over graded Lie algebras I, Math. Z., 180 (1982), 151-177.

[14] J. J. Rotman, An Introduction to Homological Algebra, Academic Press, Pure and Applied Mathematics 85, New York (1979).

[15] D. Vogan, Irreducible characters of semisimple Lie groups III. Proof of KazhdanLusztig conjecture in the integral case, Invent. Math., 71 (1983), 381-417.

Received August 11, 1987 and in revised form August 17, 1988.

University of HawaII-Kapiolani Community College

HONOLULU, HI 96816 
